\title{
Selected Scientific and Technical Contributions of Edward C. Polhamus
}

\author{
James M. Luckring $^{1}$ \\ NASA Langley Research Center, Hampton, VA, 23681
}

\begin{abstract}
Edward C. Polhamus joined the NACA Langley Research Center staff in 1944 and was active in a broad range of aerodynamic research related to high-speed aircraft technology, aerodynamic prediction methods, and cryogenic wind-tunnel development. This lecture will focus on his 'leading-edge suction analogy' for the prediction of vortex-lift effects on slender wings. Briefer treatment of his contributions to variable-sweep aircraft and cryogenic wind tunnels is also included.
\end{abstract}

\begin{tabular}{|c|c|c|c|}
\hline$A R$ & Aspect Ratio, $b^{2} / S$ & $K_{p}$ & constant, potential-flow \\
\hline$b$ & wing span & $K_{v}$ & constant, vortex flow \\
\hline$C_{D}$ & drag coefficient & $M$ & Mach number \\
\hline$C_{D, o}$ & minimum drag coefficient & $n$ & section normal force \\
\hline$\Delta C_{D}$ & $C_{D}-C_{D, o}$ & $P_{t}$ & total pressure \\
\hline$C_{L}$ & lift coefficient & $\operatorname{Re}_{c}$ & Reynolds number, $U_{\infty} c / v$ \\
\hline$C_{L L}$ & longitudinal load coefficient & $S$ & wing area \\
\hline$C_{L, p}$ & potential flow lift coefficient & $s$ & local semispan \\
\hline$C_{L, v}$ & vortex flow lift coefficient & $T_{t}$ & total temperature \\
\hline$C_{l_{p}}$ & damping in roll coefficient & $t$ & $\begin{array}{l}\text { maximum airfoil thickness, also } \\
\text { section thrust force }\end{array}$ \\
\hline$C_{m}$ & pitching moment coefficient & $U_{\infty}$ & free stream reference velocity \\
\hline$C_{N}$ & normal force coefficient & $x, y, z$ & body-axis Cartesian coordinates \\
\hline$C_{p}$ & pressure coefficient & & \\
\hline$\Delta C_{p}$ & lifting pressure coefficient & $\alpha$ & angle of attack, deg. \\
\hline$c$ & wing chord & $\varepsilon, \gamma$ & delta wing semi-apex angle, deg. \\
\hline$c_{d}$ & section drag coefficient & $\Gamma$ & circulation. \\
\hline$c_{l}$ & section lift coefficient & $\Lambda$ & sweep angle, deg. \\
\hline$c_{n}$ & section normal force coefficient & $\mu$ & viscosity \\
\hline$c_{R}$ & root chord & $v$ & kinematic viscosity, $\mu / \rho$ \\
\hline$c_{s}$ & section suction coefficient & $\rho$ & density \\
\hline$c_{t}$ & section thrust coefficient & $\phi$ & perturbation velocity potential \\
\hline \multicolumn{4}{|c|}{ Subscripts } \\
\hline le & leading edge & se & side edge \\
\hline$p$ & potential flow & $v$ & vortex flow \\
\hline ref & reference & $\infty$ & freestream reference condition \\
\hline
\end{tabular}

Abbreviations:

$\begin{array}{ll}\text { AGARD } & \text { Advisory Group for Aeronautical } \\ & \text { Research and Development } \\ \text { ATF } & \text { Advanced Tactical Fighter Program } \\ \text { CFD } & \text { Computational Fluid Dynamics }\end{array}$

LWF Lightweight Fighter Program

NACA National Advisory Committee on Aeronautics

NASA National Aeronautics and Space Administration

\footnotetext{
${ }^{1}$ Senior Research Engineer, Configuration Aerodynamics Branch, james.m.luckring@nasa.gov, AIAA Associate Fellow. 


$\begin{array}{llll}\text { DRA } & \text { Distinguished Research Associate } & \text { NATO } & \text { North Atlantic Treaty Organization } \\ \text { FX } & \text { Fighter Experimental Program } & \text { ONERA } & \text { French Aerospace Laboratory, France } \\ \text { LaRC } & \text { Langley Research Center } & \text { RAE } & \text { Royal Aircraft Establishment, UK } \\ \text { LMAL } & \begin{array}{l}\text { Langley Memorial Aeronautical } \\ \text { Laboratory }\end{array} & \text { TFX } & \text { Tactical Fighter Experimental Program } \\ & & & \end{array}$

\section{Introduction}

$\mathrm{E}$ dward C. Polhamus spent his career working for the NACA and NASA at the Langley Research Center in Hampton, Virginia. Having come to the lab in 1944, he was able to contribute aerodynamic research during an unusually rich era of aircraft development for the United States. Polhamus' career was focused, to a large degree, on the configuration aerodynamics of aircraft with high-speed capability. Much of his work was performed at subsonic to low transonic speeds, studying aerodynamic characteristics associated with the evolution of swept-wing and slender-wing configurations. Polhamus' interest spanned performance and maneuver aerodynamics, including separated flow and Reynolds number effects. His research included a mixture of fundamental aerodynamic studies, such as with delta wings, and configuration aerodynamic studies that related to national programs.

Three topics from Polhamus' career have been chosen to highlight in this paper. The first is the development of the leading-edge suction analogy for the prediction of separation-induced vortex flows and vortex-lift increments on slender wings. This work contributed to the practical use of vortex lift for slender-wing aircraft. The second is the development of the outboard-pivot concept for variable-sweep aircraft. This work made variable sweep a practical consideration for high-speed aircraft. The third is the development of the 0.3-m Transonic Cryogenic Tunnel. This work demonstrated that the cryogenic concept was practical for obtaining Reynolds number effects and high Reynolds number data.

The majority of this paper is dedicated to the suction analogy and includes some of the history for the evolution of interests in slender-wing vortex flows. Both the variable-sweep and the Reynolds number research topics are presented with much less detail in this paper. The material selected helps to establish the scope of Polhamus' contributions, and each of these topics could benefit from a separate and dedicated publication. Some background material is presented first.

\section{Background}

Edward C. Polhamus was born in 1923 and was a native of Washington D.C. where his father was a tool and die maker. After one quarter at Virginia Polytechnic and State University, he chose to attend the University of Maryland to study engineering. Academic programs had been accelerated in association with World War II, and he completed his undergraduate studies in three years, earning a Bachelor of Science degree. He was hired by the National Advisory Committee on Aeronautics, NACA, and reported to the Langley Memorial Aeronautical Laboratory, LMAL, in July of 1944. A photograph of a young Edward C. (Eddie) Polhamus is shown in Figure 1 near an apartment complex where engineers who worked at NACA Langley lived. With the exception of a brief assignment at NACA headquarters in the early 1950s, Polhamus spent his entire career at NACA/NASA Langley conducting and guiding configuration aerodynamics research at subsonic and transonic speeds. His focus was on slender airframe aerodynamics, and his primary tools were the wind tunnels at Langley. Polhamus combined experimental aerodynamics with a keen intuition for aerodynamic flows; he was an analyst. He spent about half of his career as a practicing researcher and half leading his research branch. After retirement in the summer of 1981, he continued pursuing his research interests as a Distinguished Research Associate at NASA Langley through the summer of 1996.

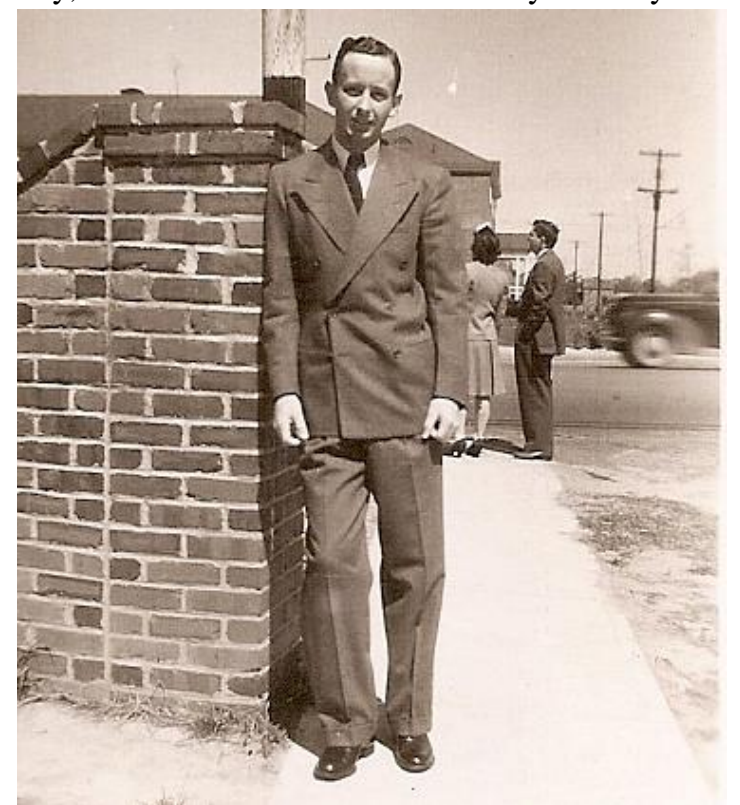

Figure 1. Edward C. Polhamus, 1946. 
At the time Polhamus began his career, the country was focused on ending World War II, and, in the process, was learning much about the advanced German aircraft programs. It was becoming clear that the evolution of fighter aircraft for high-speed flight was at its beginning, and that new concepts such as the swept wing, the slender delta wing, jet propulsion, and more would revolutionize the fighter aircraft airframe. The slender wing concepts were also recognized for contributing to the possibility of a supersonic transport. The onset of the cold war accelerated the national commitment to advanced aircraft, and a remarkably rich aircraft development era followed. During Polhamus' career, a succession of national programs revolutionized slender wing aircraft with many airframes, the likes of which had not been seen before. The slender airframe work was also happening at a very fast pace. From the F-100 to the F-18 the United States deployed approximately 14 unique slender airframe aircraft in slightly more than 25 years, for approximately 1 new airframe every 22 months. Additional research and prototype aircraft were also developed. There was very close coordination among the U.S. national programs, U.S. airframe companies and the national laboratories. Much of Polhamus' work was related to these national programs. In addition, the slender-wing supersonic transport aircraft Concorde was created during this same time period, along with other European slender aircraft as well as a suite of Soviet slender airframes.

A nominal timeline is presented in Figure 2 to put in perspective Polhamus' tenure at Langley, selected aircraft development programs, and global events that were driving the aircraft programs. Polhamus' career with the NACA and NASA is summarized at the top of the chart. The slender-wing development programs in the middle of the chart each span the beginning of focused study/development to a first flight date. The U.S. programs shown are (i) the Century Series (F-100 through F-106), (ii) the Tactical Fighter Experimental program (TFX, F-111), (iii) the Fighter Experimental program (FX, F-15), (iv) the Lightweight Fighter program (LWF, F-16), and (v) the Advanced Tactical Fighter program (ATF, F-22). The British-French Concorde program is also shown, and a number of other significant slender-wing aircraft not shown on the chart were also developed during this same time period. Some major global events that drove these aircraft development programs are shown at the bottom of the chart. The work Polhamus performed on vortex lift, variable sweep, and high Reynolds number aerodynamics contributed to many of these national programs as well as some others not mentioned.

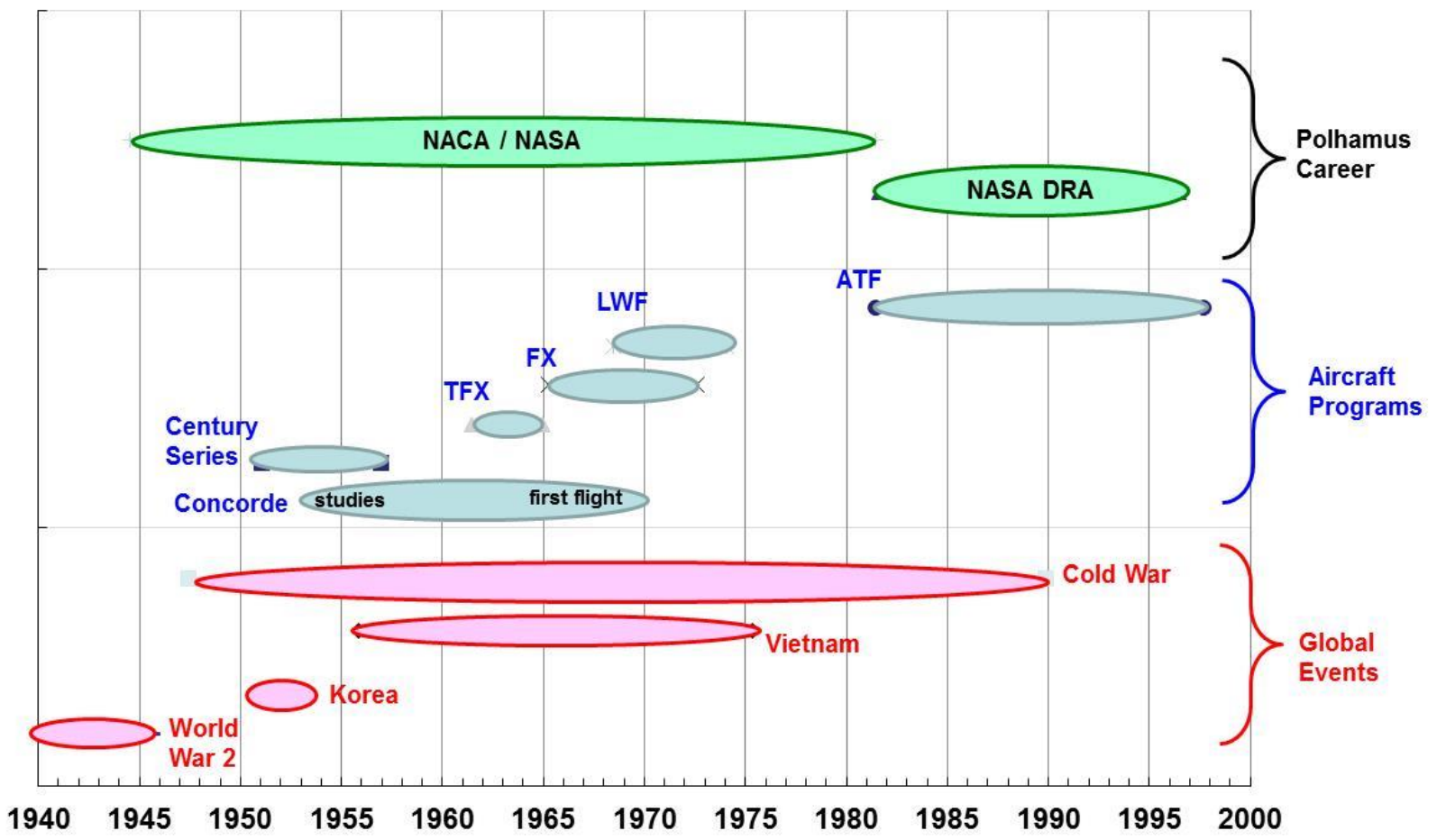

Figure 2. Timeline.

The central paper leading to this lecture was published by Polhamus ${ }^{1}$ in the Journal of Aircraft in 1971, "Predictions of Vortex-Lift Characteristics by a Leading-Edge Suction Analogy." In discussing this work, it is useful to understand some of the origins of slender-wing vortex-flow aerodynamics and their role in the development of slender-wing aircraft concepts. This will take us to the end of World War II and the beginning of Polhamus' career. 


\section{Vortex-Lift Research}

The experimental discovery of vortex lift occurred at NACA Langley shortly after Polhamus arrived at the laboratory and is briefly reviewed below. Next the evolution of theoretical modeling of this flow is summarized. These works provide the background for Polhamus' accomplishment with his leading-edge suction analogy, which is then presented.

\section{A. Experimental Discovery of Vortex Lift}

The thin delta wing, combined with jet propulsion, had been identified as a fighter vehicle concept that offered promise for supersonic flight capability. However, these wings did not necessarily offer acceptable lowspeed aerodynamics due to their low aspect ratio and small leading-edge radius. For example, the low liftcurve slope associated with the low aspect ratio wing could result in inadequate lift at typical speeds for landing. An unusual full-scale deltawing configuration, developed by Dr. Alexander Lippisch, had been discovered at the Munich Prein airport as World War II was ending (Figure 3), and it was decided to study the low-speed aerodynamics of this vehicle. The vehicle was known as the Darmstadt-München-1 (or DM-1), and it was shipped to NACA Langley for testing in the LMAL 30-by-60 foot full scale tunnel (Figure 4). The tests were performed in 1946 and reported by Wilson and Lovell. ${ }^{2}$

The DM-1 differed from the high-speed delta wing planning of that time in that it was thick and had very blunt leading edges. Initial test results showed an unanticipated low angle of attack for wing stall, with a corresponding low maximum lift coefficient. Earlier tests of subscale models had not shown this feature, and subsequent testing of a new subscale model of the DM-1 revealed a laminar separation at the leading edge, with subsequent vortical flow over the wing. At the low Reynolds numbers of these tests, this flow produced high lift coefficients at high angles of attack. It was then reasoned that a sharp

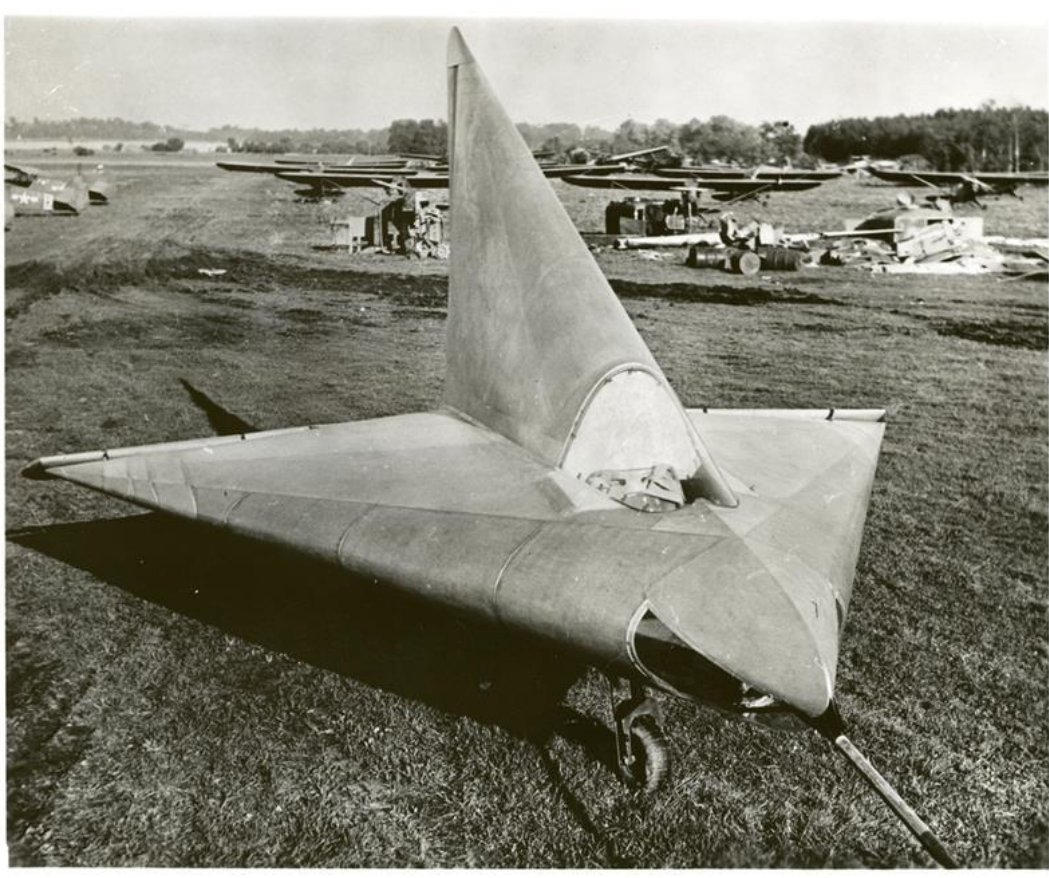

(a) DM-1 vehicle

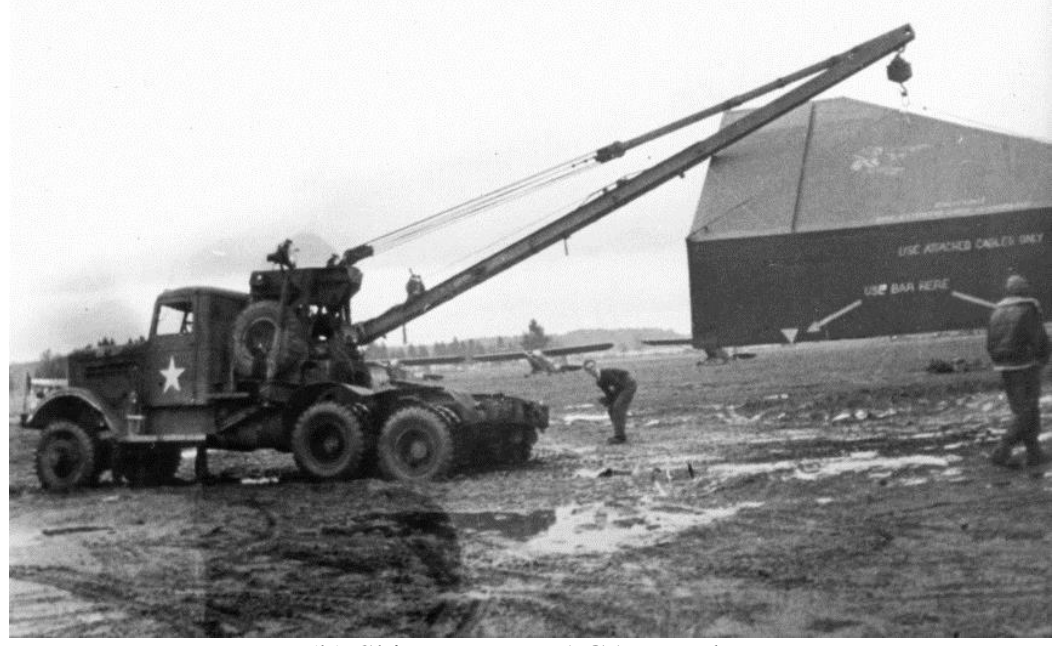

(b) Shipment to NACA Langley

Figure 3. Lippisch DM-1 glider, 1945.

leading edge could force the leading-edge vortex flow to occur, even at the high Reynolds numbers of the full scale DM-1 vehicle. The DM-1 was modified to incorporate a sharp leading-edge strip, as shown in Figure 5, and the 
subsequent testing produced large lift increments, compared to the clean configuration, due to the formation of the separation-induced leading-edge vortices over the wing. An example of the forces and moments from the Wilson and Lovell report is shown in Figure 6. Wilson and Lovell attributed the lift increments to the formation of leadingedge vortices, and a second figure from their report with this interpretation is reproduced in Figure 7. Dr. Samuel Katzoff of NACA Langley contributed to the early and qualitative interpretations of these vortical flows, and the Wilson and Lovell results established the connection between the high angle-of-attack lift increments and leadingedge vortex flows.

This was the first interpretation of separationinduced leading-edge vortex-lift effects. However, the work and report remained classified for some time and so was not known to the broader slender wing community that was developing. Some additional details of the experiments have been given by Chambers, ${ }^{3}$ and additional comments on the discovery of vortex lift have been given by Polhamus. $^{4}$ In this report, Polhamus also summarized the controlled separation concept as an adjunct to the more traditional design principles that are anchored in sustaining attached flow. The controlled separation, as in the formation of a leadingedge vortex, offered a means of compromise between the highspeed cruise design interests (attached flow) and the lower speed high angle-of-attack maneuver and take-off/landing interests (vortex flow).

These vortex flows could at least be studied experimentally in the course of developing the new generation of slender-wing aircraft. However, there were no theories to predict the high angleof-attack vortex-flow aerodynamics, and this need led to an evolution of theoretical methods, including the suction analogy.

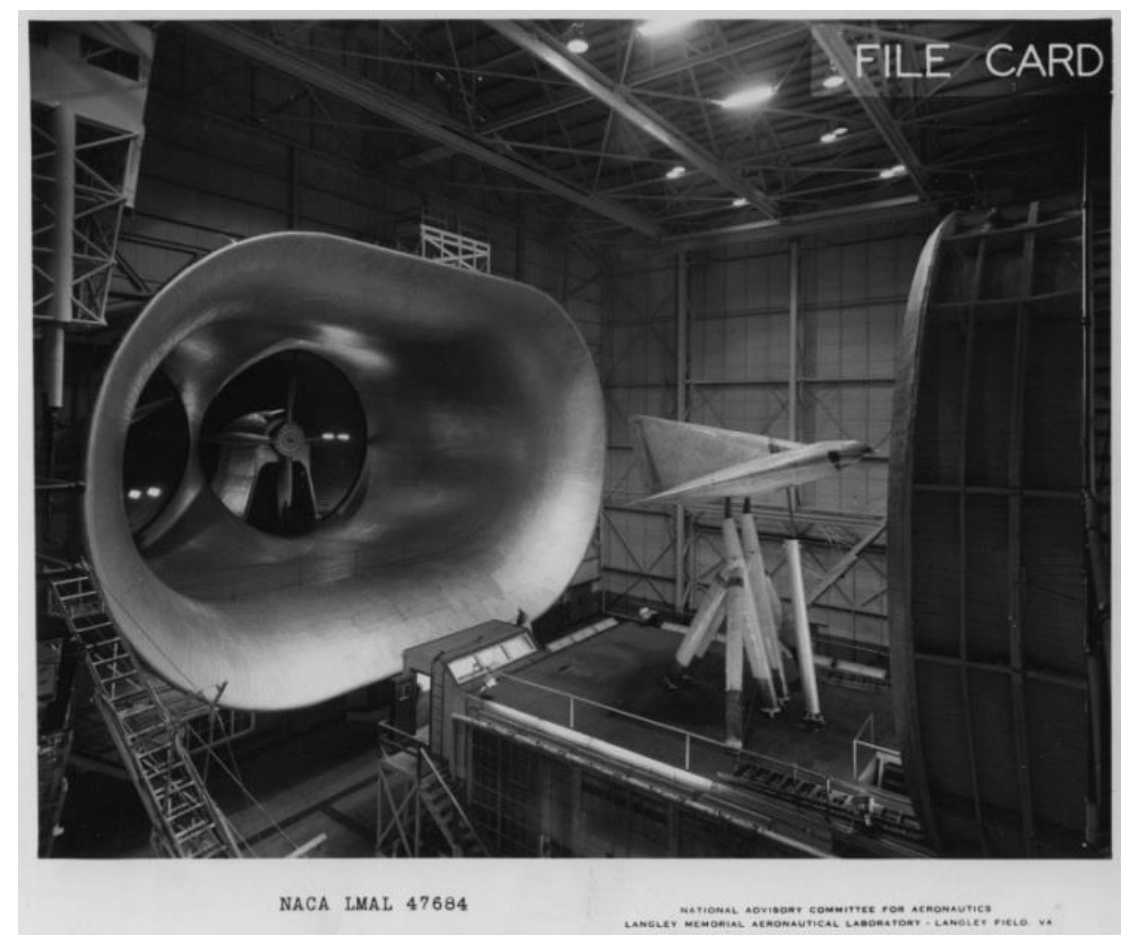

Figure 4. DM-1 glider test in LMAL 30- by 60-Foot Full-Scale Tunnel. 1946.

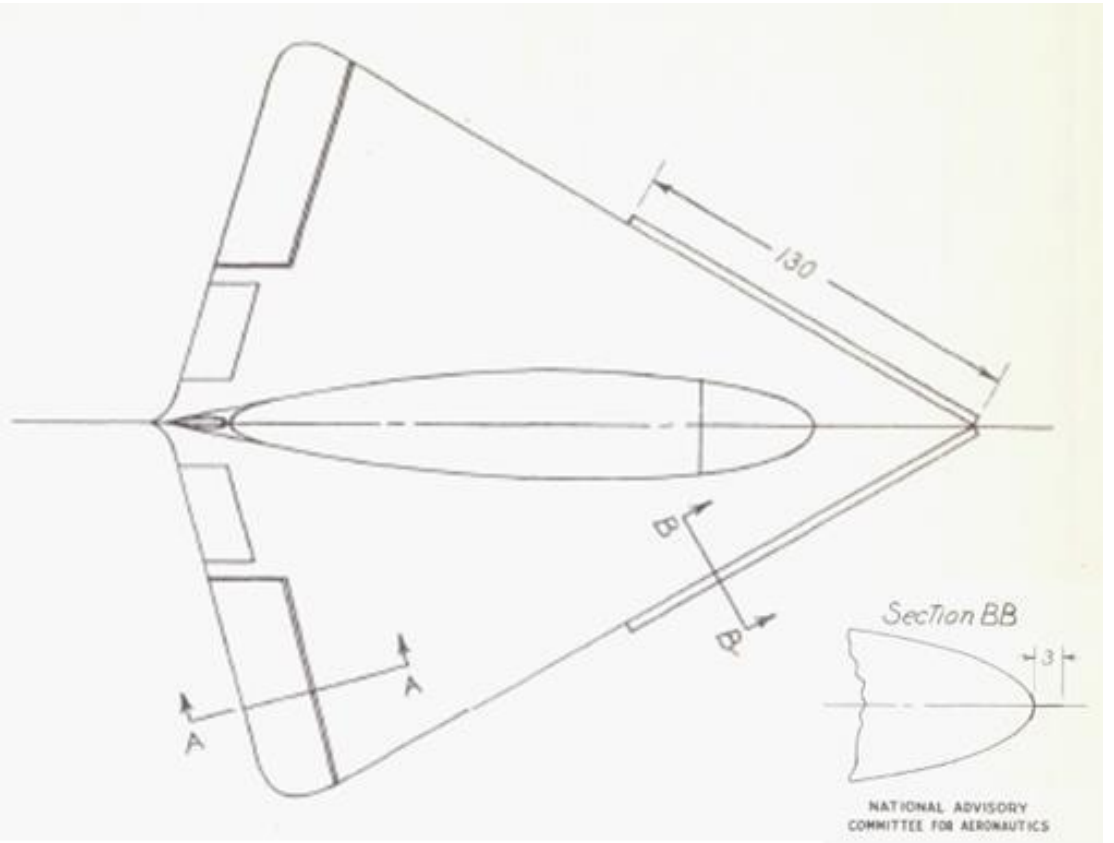

Figure 5. Drawing of DM-1 glider with sharp leading-edge strip. From Wilson and Lovell. ${ }^{2}$ 


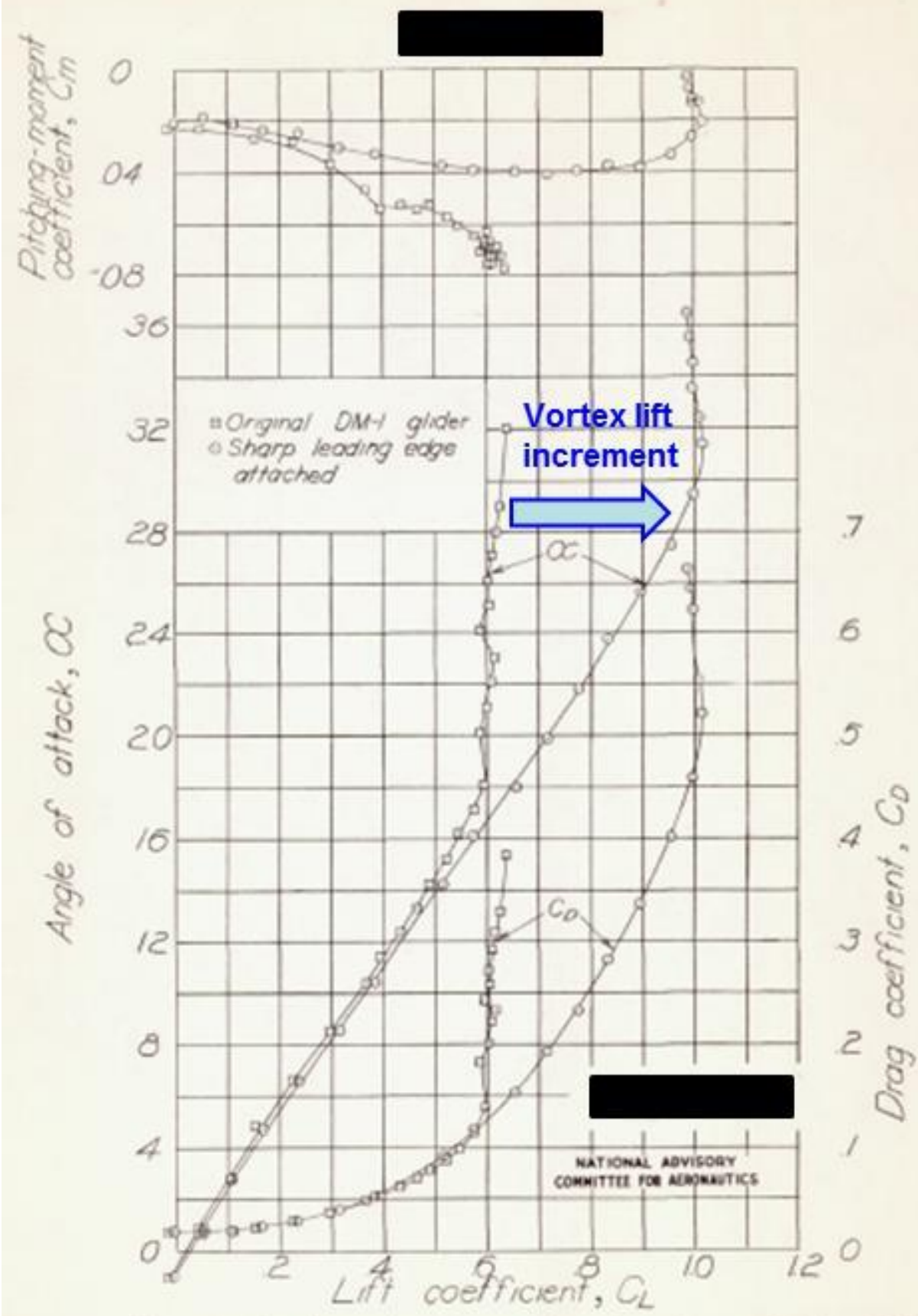

Figure 3-Aeradynamic characteristic of the DM-1 gilder with and without sharp leading edges.

Figure 6. DM-1 forces and moments. From Wilson and Lovell. ${ }^{2}$ 


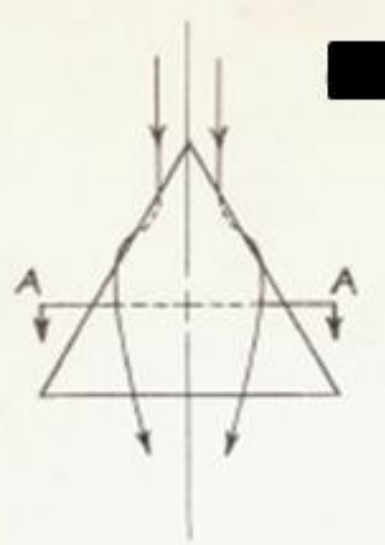

(a) Elements of flow over

a triangular wing

having a rounded

leading edige of high

Reynolds numbers.

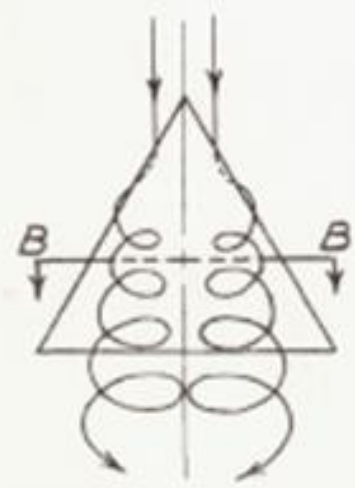

(c) Elements of flow over

a trianguiar wing at low

Reynolds numbers or

over a triangular wing

having a sharp leading edge.

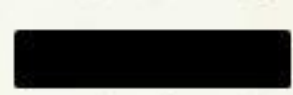

(b) Elements of the vertical component of flow over section $A A$.

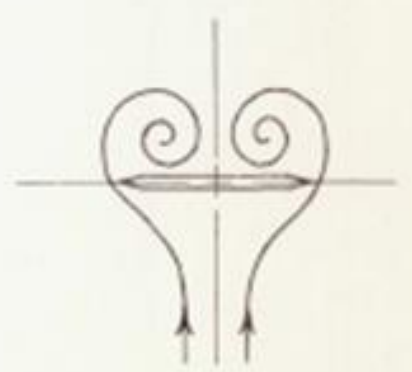

(d) Elements of the vertical component of flow over section $B B$.

NATIONAL ADVISORY COMMITTEE FOQ AERONAUTICS

Figure 5.- Diagrams of the flow over triangular wings.

Figure 7. DM-1 forces and moments. From Wilson and Lovell. ${ }^{2}$ 


\section{B. Evolution of Theoretical Models: Conical Flow}

The initial evolution of theoretical methods to predict slender wing aerodynamics and separation-induced leading-edge vortex effects is shown in Figure 8. All of these methods were based on conical flow assumptions so that crossflow plane solutions could be achieved through the use of complex variables. All of this work predates the suction analogy.

Jones $^{5}$ first developed the attached flow slender wing solutions in 1946. His attached flow theory provides the baseline from which to judge the separated flow theories. The first solution to include the effect of a leading-edge vortex was developed by Legender ${ }^{6}$ in 1952 by including the effects of concentrated leading-edge vortices over the slender wing in his solution. His results showed a similarity form to the solution in terms of the ratio of the angle of attack and the delta wing semi-apex angle. Brown and Michael $^{7}$ extended this model to include a flat feeding sheet to approximate the vorticity shedding from the leading edge and forming into the concentrated vortex. Their model demonstrated that the solution took on a superposition of the Jones slender wing flow plus higher order terms (h.o.t.) that accounted for the vortex effects. Mangler and Smith ${ }^{8}$ introduced an analytical curved feeding sheet as a better approximation from the Brown and Michael work, and Smith $^{9}$ developed a segmented feeding sheet as further improvement. The collective work of Figure 8 spanned 20 years, and spanwise pressure distributions from the Smith theory resembled the expected trend for a slender wing with separation-induced leading-edge vortex flow. As would be expected, the solution differs significantly from the Jones attached flow result.

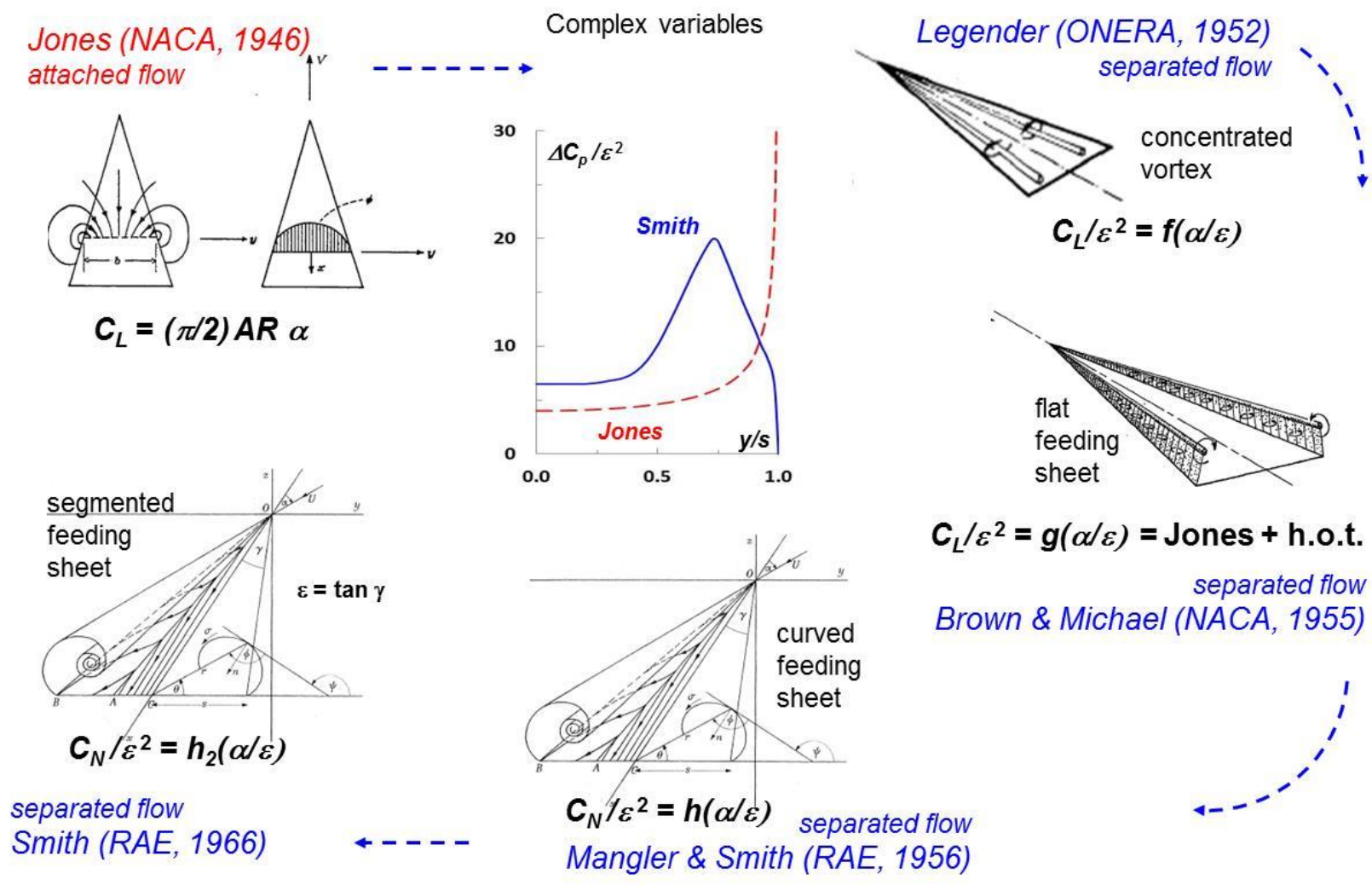

Figure 8. Early slender wing and vortex- lift theories. Conical flow.

A timeline for vortex-lift prediction publications, including these conical flow theories, is shown in Figure 9. The figure also includes the Wilson and Lovel ${ }^{2}$ experimental publication, for reference, as well as several publications by Polhamus and his coworkers that will be addressed later. The time frame for the DM-1 tests as well as the airframe development programs for the Century Series aircraft, the Concorde, and the Lightweight Fighter aircraft are also shown at the bottom of the figure.

With the exception of the Brown and Michael $^{7}$ work, all the theoretical advances were performed in Europe, and this European work was motivated by the development activities for the Concorde supersonic transport. The Concorde was being designed with a slender ogee-planform wing for efficient supersonic cruise, and would rely on separation-induced leading-edge vortex-lift increments at takeoff and landing conditions. These advances in Europe were remarkable, and since the discovery of vortex lift in the (classified) DM-1 work, followed by the Brown and Michael theory, the US had fallen more than 10 years behind in the theoretical modeling of these flows. 


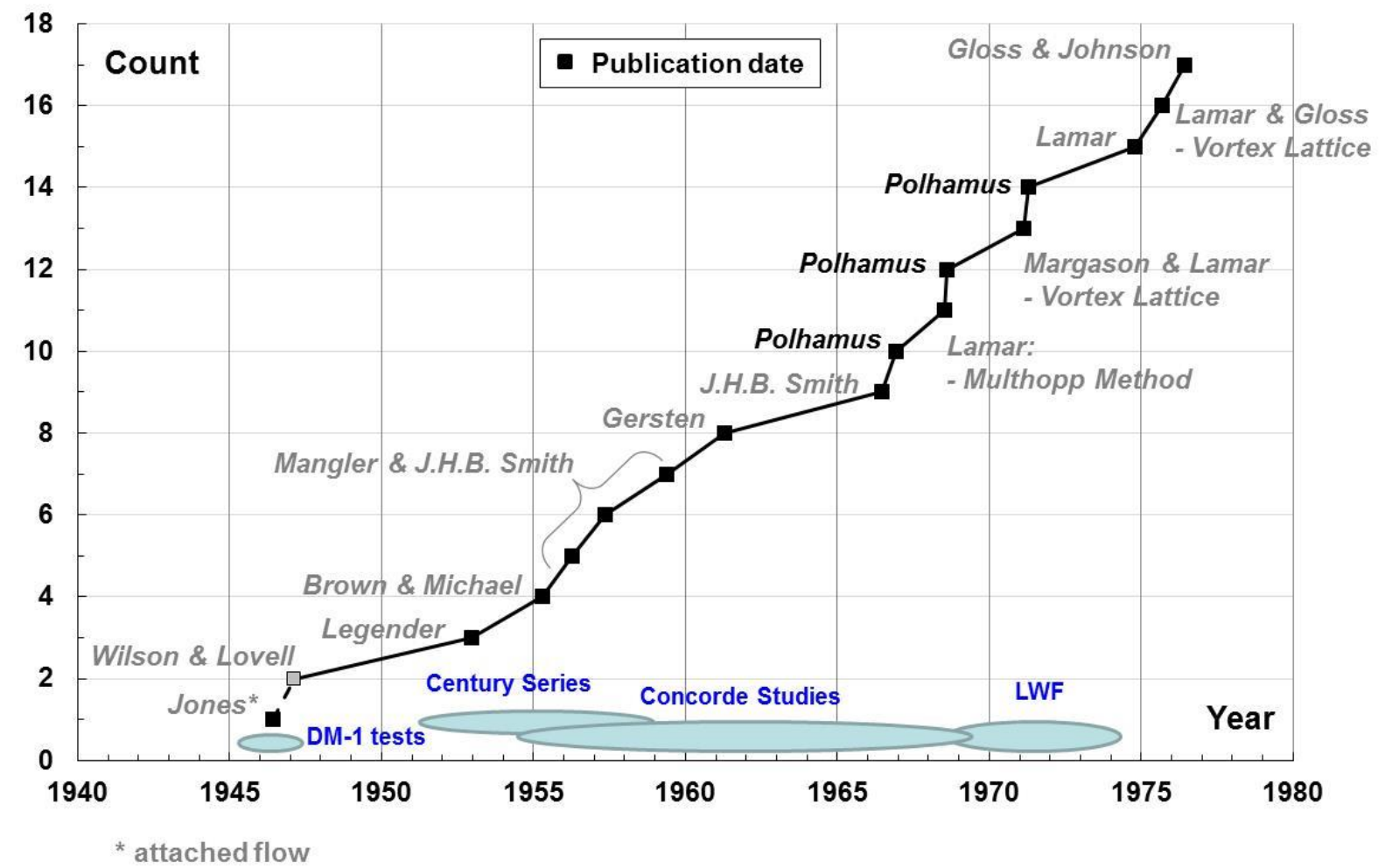

Figure 9. Vortex lift prediction papers.

\section{Polhamus Suction Analogy}

The conical flow theories just discussed were all directed at obtaining spanwise pressure distributions that included effects of the separation-induced leading-edge vortices on the slender wing flow. However, by the very nature of these formulations, there was no representation of three-dimensional effects. A summary of some of these effects is shown in Figure 10, derived from Polhamus, ${ }^{10}$ for simple delta wings with a spiral vortex sheet separating from a sharp leading edge. The flow has a singularity in the pressure distributions at the apex and the wing loading must satisfy the trailing-edge Kutta condition. A conical flow would have pressures that were constant along rays emanating from the apex, and there is no region of a three-dimensional delta wing, or other slender wing, that exhibits this feature. As a consequence, the conical theories did not model finite aspect ratio effects on lift as shown on the right portion of the figure for a fixed angle of attack. The theory that is closest to the experimental data was due to Gersten, ${ }^{11}$ and this was an approximate three-dimensional approach. However, the Gersten model only accounted for about half the vortex-lift increment as referenced to the attached flow linear theory shown.

Polhamus approached this problem from a three-dimensional aerodynamic perspective and had two insights for what became the leading-edge suction analogy. The first insight regarded forces from the wing leading-edge suction and the second regarded high angle-of-attack boundary conditions.

To explain the leading-edge suction insight, it is first useful to recall some airfoil properties for an ideal fluid, Figure 11. A Joukowski airfoil is used for convenience, and the figure shows the section normal and thrust coefficient components of the sectional lift coefficient; in these flows the sectional drag coefficient is exactly equal to zero. The force coefficients (including the leading-edge thrust) vary slightly with thickness, and, in the limit of a flat plate, the thrust coefficient is sustained, now in association with the leading-edge singularity. In the case of flow about a wing, the leading-edge thrust coefficient is a component of the leading-edge suction coefficient, and this relationship is shown on the left portion of Figure 12 for a delta wing. 


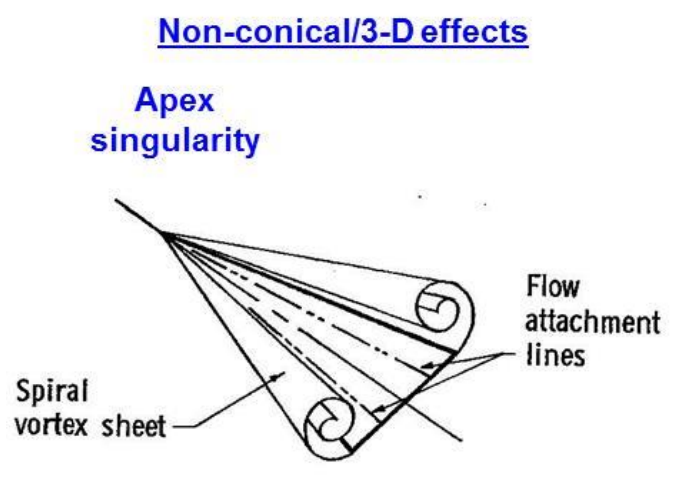

Trailing-edge Kutta condition

Polhamus bridged this gap:

1. Connection of vortex lift with leading-edge suction

2. Implementation with high angleof-attack boundary conditions

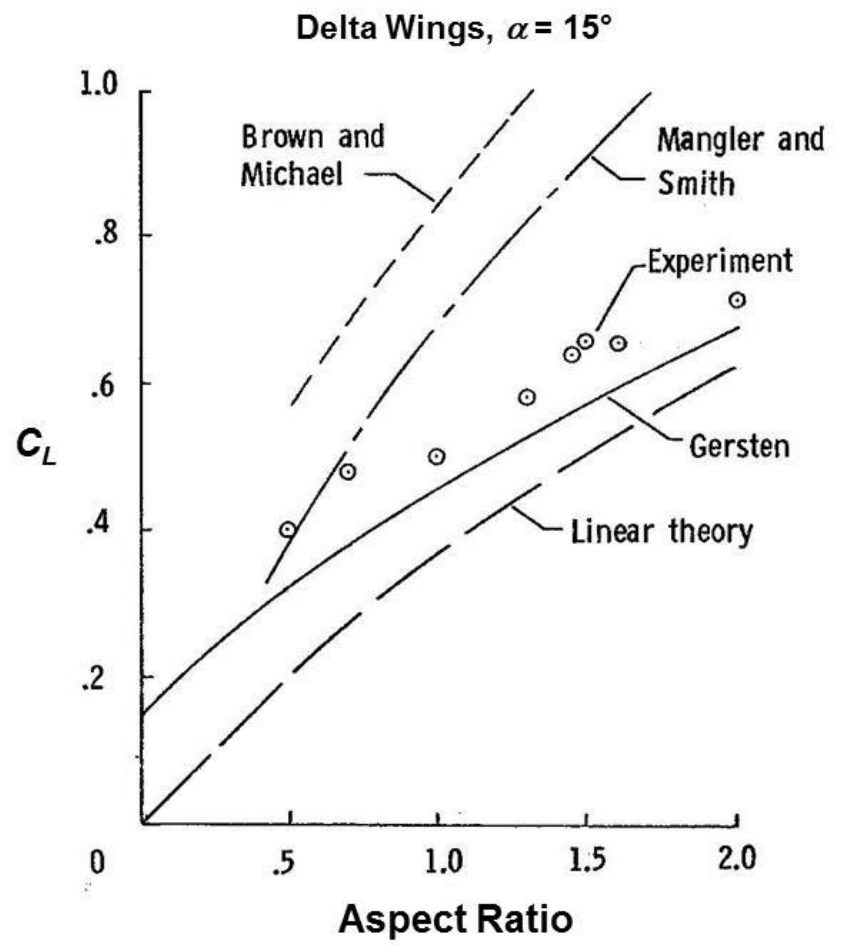

Figure 10. Three-dimensional effects on vortex lift. Derived from Polhamus. ${ }^{10}$

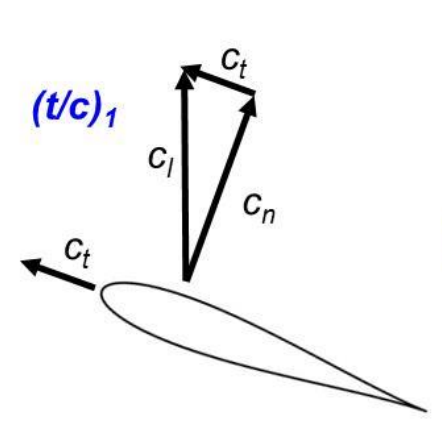

2-D flow

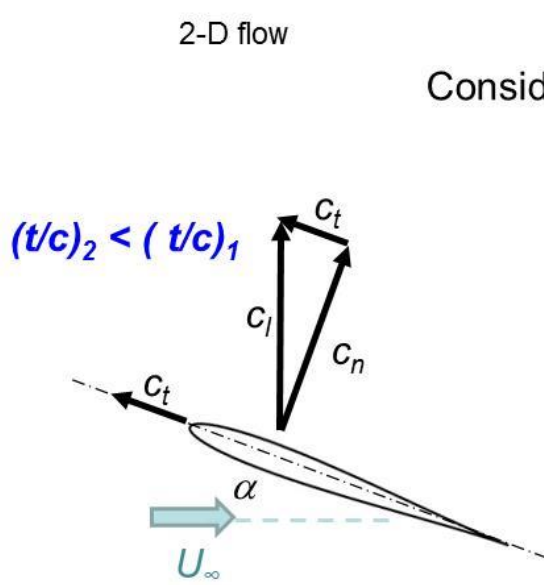

As t/c diminishes:

- Leading edge radius decreases

- Leading edge velocities increase / pressures decrease

- Thrust force approaches a finite limit

Drag is exactly zero

Thrust force created by leading-edge suction

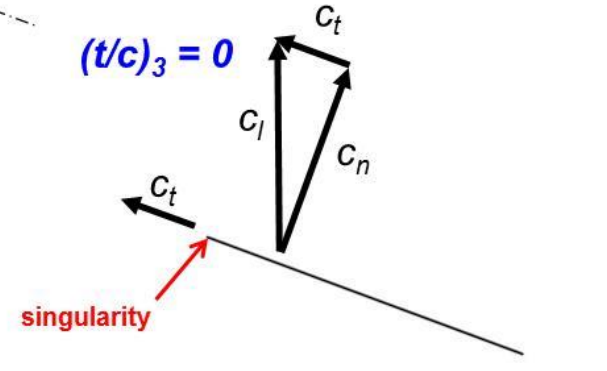

Figure 11. Conservation of leading-edge thrust. 


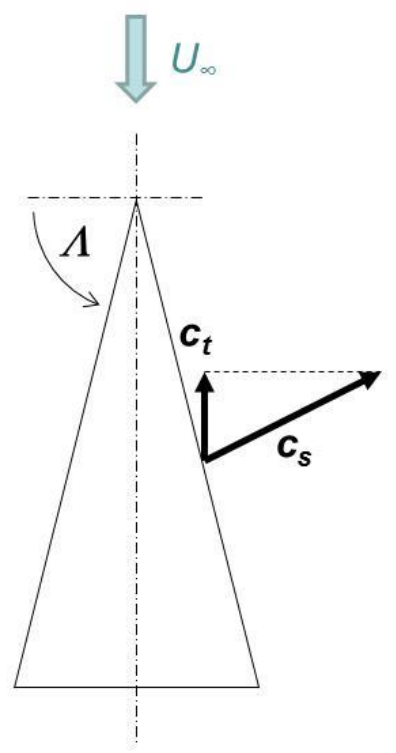

Plane normal to leading edge
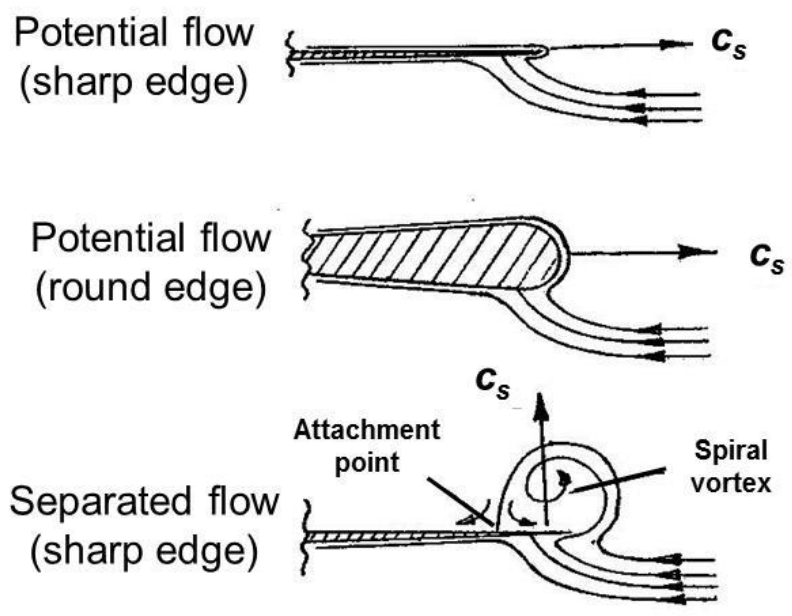

\section{Reasoning was for low angles of attack, when vortex first forms near the leading edge}

Figure 12. Suction analogy.

Cross sections normal to the leading edge are shown on the right portion of the figure for three flow conditions. The top sketch is for attached potential flow about a sharp-edged wing and shows the sectional leading-edge suction coefficient associated with the leading-edge singularity. The middle sketch is for attached potential flow about a blunt-edged wing, and again shows the sectional leading-edge suction coefficient. This suction force will differ very little from the sharp-edged case. The bottom sketch is for the separated vortex flow about the sharp edge. Polhamus drew an analogy between the suction pressures required to maintain the flow about the leading-edge vortex and those required to maintain the potential flow about the leading edge. The reasoning was that at angles of attack where the vortex first formed, and the flow reattached on the upper surface, the suction pressures, and hence suction force coefficient, would be about the same as for the attached potential flow case. For the reattached vortex flow, the suction force coefficient has been reoriented from acting in the plane of the wing to acting in the plane normal to the wing upper surface. This became the leading-edge suction analogy.

The interest in these vortex flows was for the higher angles of attack associated with maneuver and takeoff/landing conditions, and Polhamus' second insight for the suction analogy was to account for the exact boundary conditions and force vector orientations with respect to angle of attack. These basic ideas are reviewed in Figure 13 for the two dimensional flow of an ideal fluid about a flat plate. The lifting force is manifested through the circulation, $\Gamma$, and by simply considering a single-element panel, the circulation strength $\Gamma$ will be proportional to $\sin (\alpha)$, and the body axis forces will be proportional as:

$$
\begin{gathered}
n \sim U_{\infty} \cos (\alpha) \sin (\alpha) \\
t \sim U_{\infty} \sin ^{2}(\alpha)
\end{gathered}
$$

These relationships account for the wing and force vector orientations exactly with respect to the angle of attack. The problem is still linear, but now in terms of $\sin (\alpha)$. 


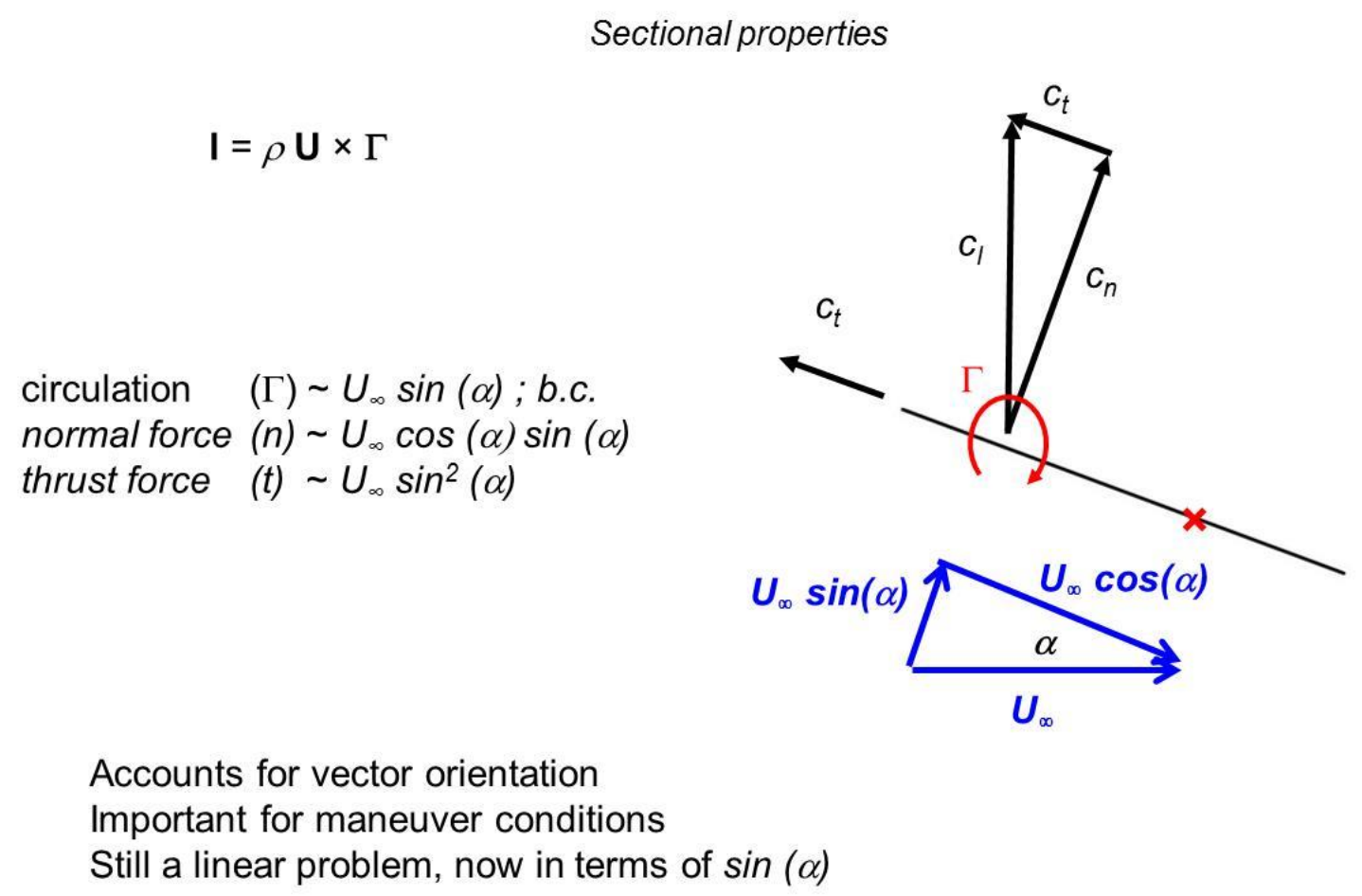

Figure 13. High angle-of-attack boundary conditions.

The same relationships hold for wings, and the approach taken was to perform the wing computations with linear methods, such as a vortex lattice. The basic approach is shown in Figure 14. The constants of proportionality for the attached flow $\left(K_{p}\right)$ and vortex flow $\left(K_{v}\right)$ normal forces could be extracted from the basic solution, and with the high angle-of-attack effects the solution took the form:

$$
\begin{gathered}
C_{N, p}=K_{p} \cos (\alpha) \sin (\alpha) \\
C_{N, v}=K_{v} \sin ^{2}(\alpha)
\end{gathered}
$$

For the lift components we have

$$
\begin{aligned}
& C_{L, p}=K_{p} \cos ^{2}(\alpha) \sin (\alpha) \\
& C_{L, v}=K_{v} \sin ^{2}(\alpha) \cos (\alpha)
\end{aligned}
$$

and hence

$$
C_{L}=C_{L, p}+C_{L, v}=K_{p} \cos ^{2}(\alpha) \sin (\alpha)+K_{v} \sin ^{2}(\alpha) \cos (\alpha)
$$

Since the leading-edge thrust is no longer manifested in the plane of the wing we also have

$$
C_{D}=C_{L} \tan (\alpha)
$$

and in this relationship the lift coefficient and angle of attack include the vortex-lift effects. The basic form of the lift variation with angle of attack is shown on the left portion of Figure 14. The attached flow lift comes from the wing normal forces, and the vortex lift comes from the suction analogy. The nonlinear trends in both come from the exact high angle-of-attack boundary conditions and force vector orientations inherent to the suction analogy as set out by Polhamus. 


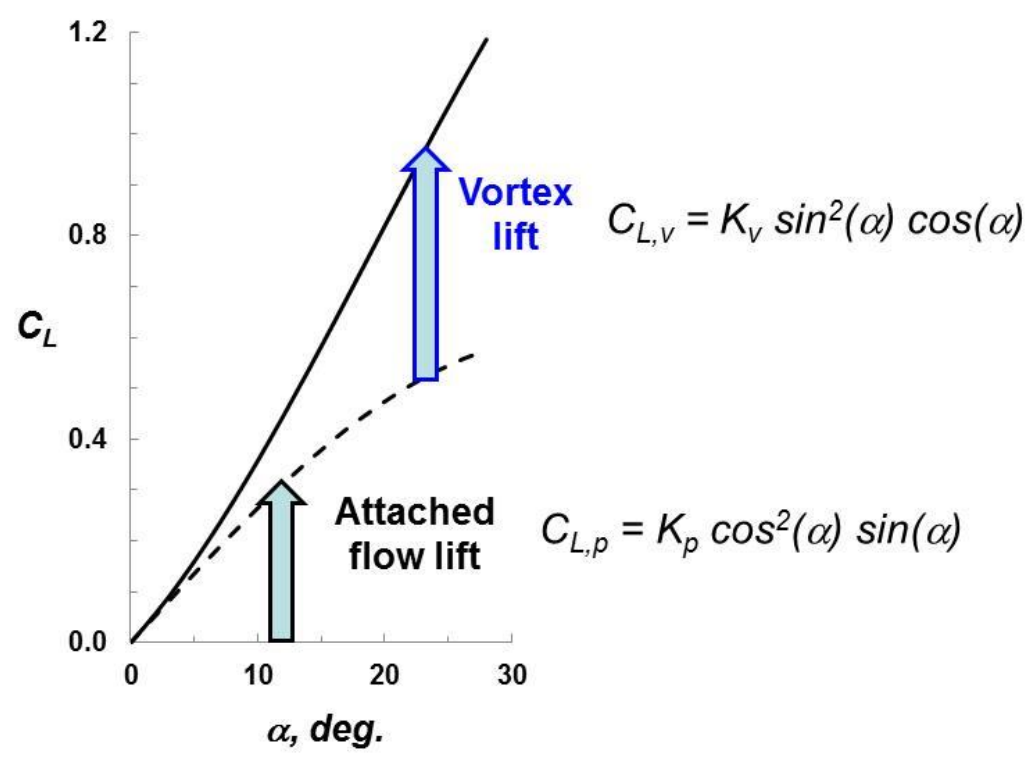

$$
\begin{aligned}
& C_{N, p}=K_{p} \cos (\alpha) \sin (\alpha) \\
& C_{N, v}=K_{V} \sin ^{2}(\alpha)
\end{aligned}
$$

$\alpha$, deg.

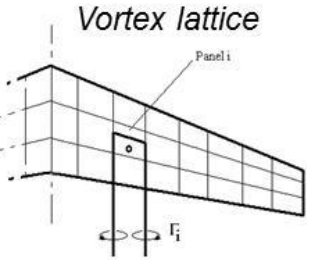

Linear system

$\mathbf{A} \Gamma \mathbf{=} \mathbf{b}$

$K_{p}=f(\Gamma)$

$K_{v}=g(\Gamma)$

$$
C_{L}=C_{L, p}+C_{L, v}=K_{p} \cos ^{2}(\alpha) \sin (\alpha)+K_{v} \sin ^{2}(\alpha) \cos (\alpha)
$$

Figure 14. Vortex lift, leading-edge suction analogy.

Polhamus ${ }^{10}$ published this approach in 1966, and included initial assessments of the suction analogy formulation against data for delta wings. The effect of aspect ratio, at a fixed angle of attack, is repeated from Figure 10 in Figure 15, but now with the suction analogy result included. These results were very surprisingly encouraging, and were a significant improvement as contrasted with the estimates from the conical flow formulations. Lift coefficient trends with angle of attack for delta wings of varying aspect ratio were compared between the suction analogy and experiment, and Polhamus' findings are repeated in Figure 16. The correlation is extremely good up to high angles of attack. The results from Polhamus' original suction analogy publication ${ }^{10}$ were the first accurate and general predictions of vortex lift for wings.

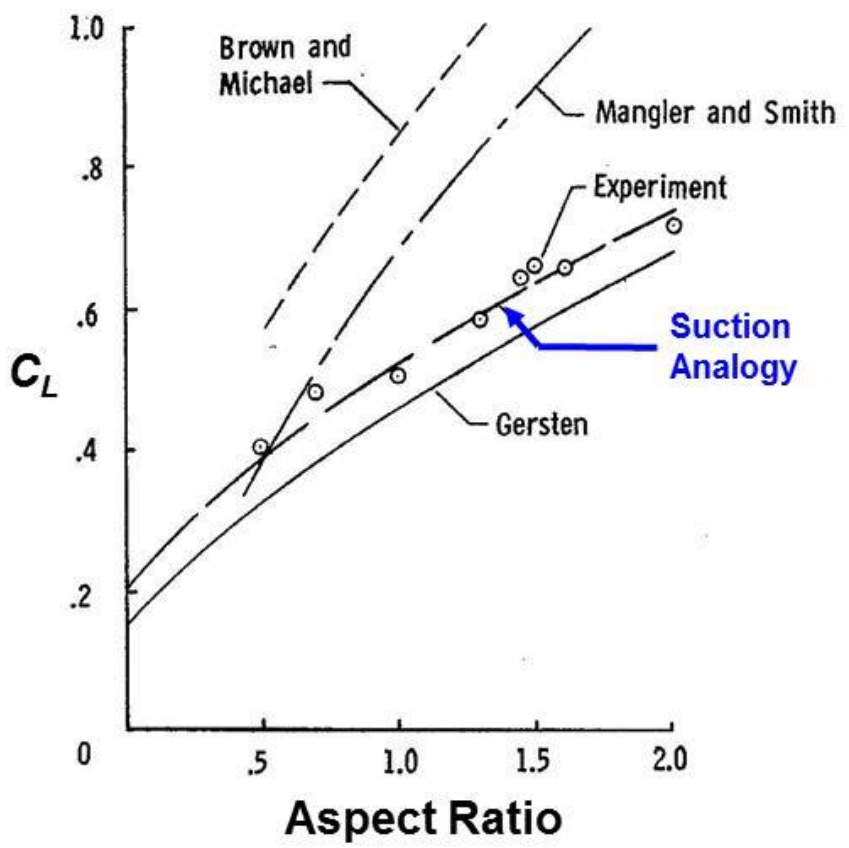

Figure 15. Application of suction analogy to delta wings, $\alpha=15^{\circ}$. From Polhamus. ${ }^{10}$ 
AIAA $34^{\text {th }}$ Applied Aerodynamics Conference Washington, DC
AIAA 2016-xxxx

Historically Significant Papers
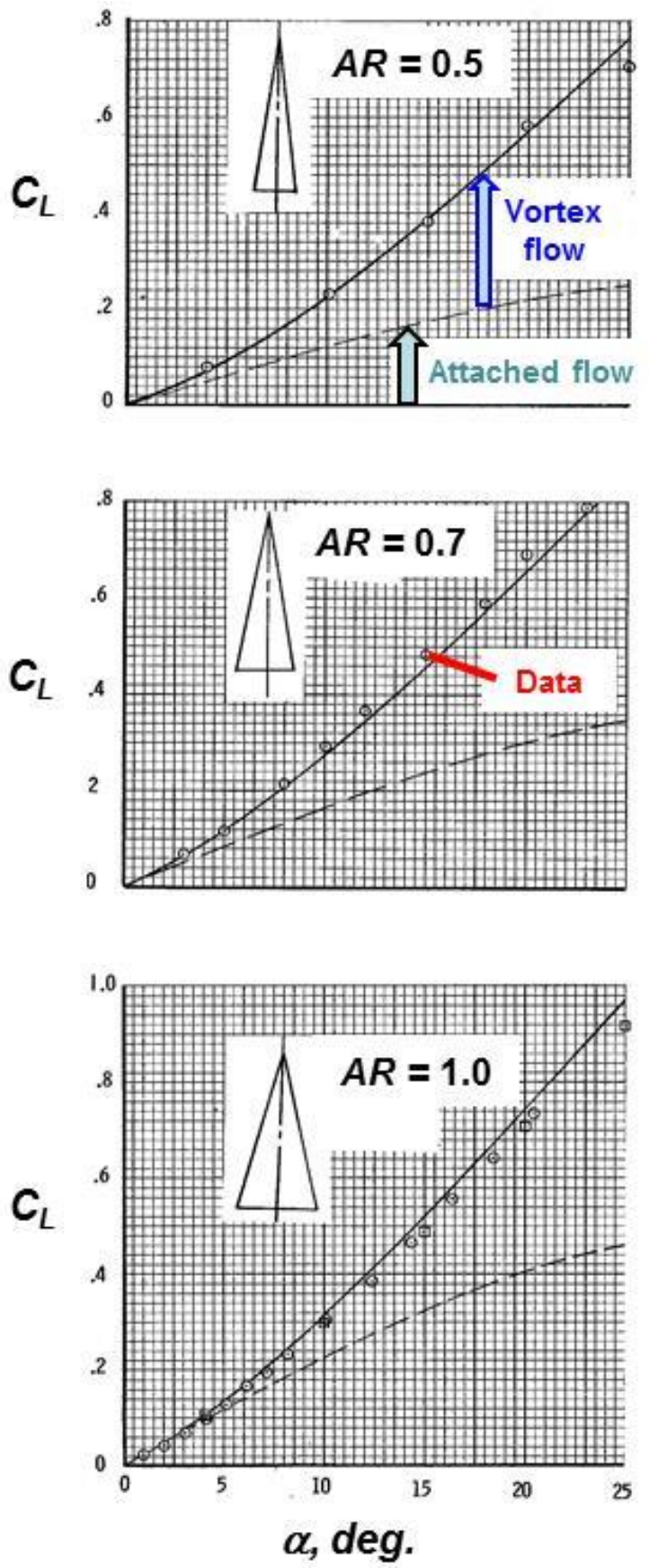
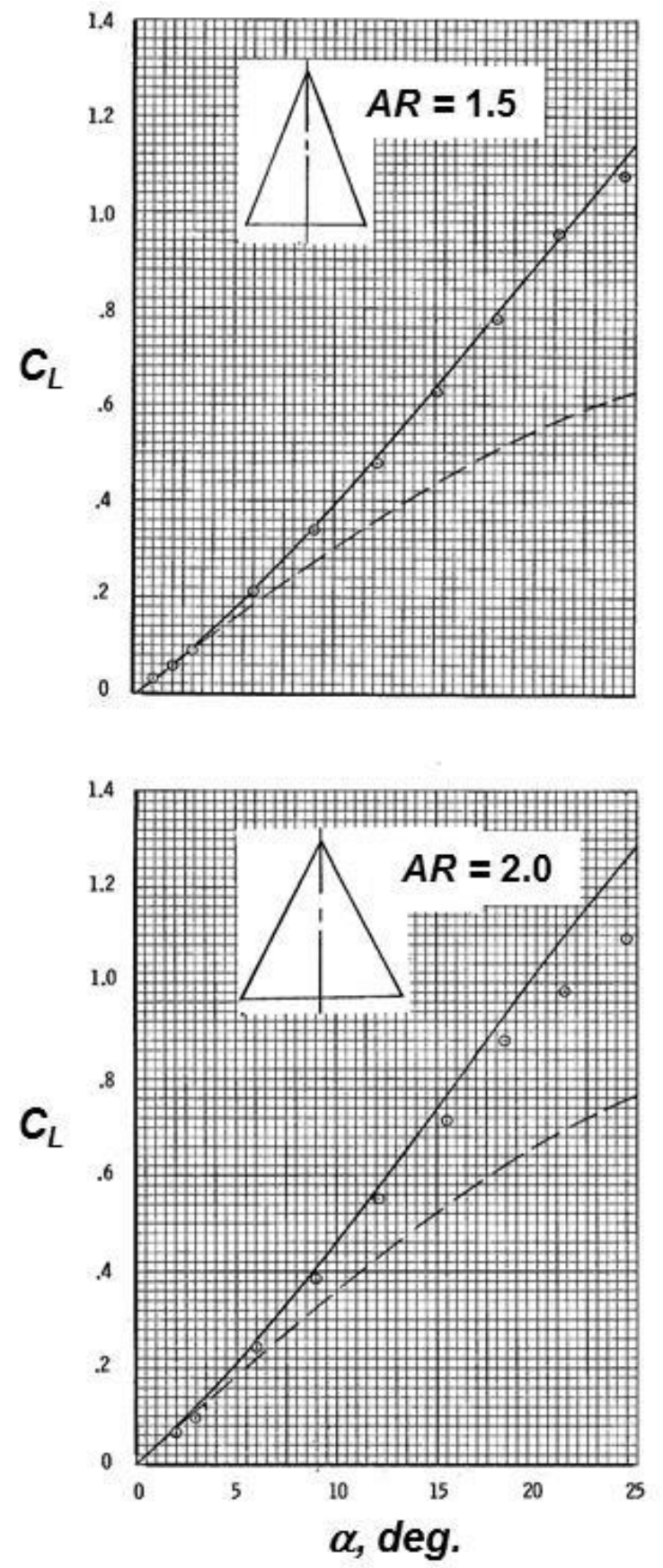

Figure 16. Application of suction analogy to delta wings. From Polhamus. ${ }^{10}$ 


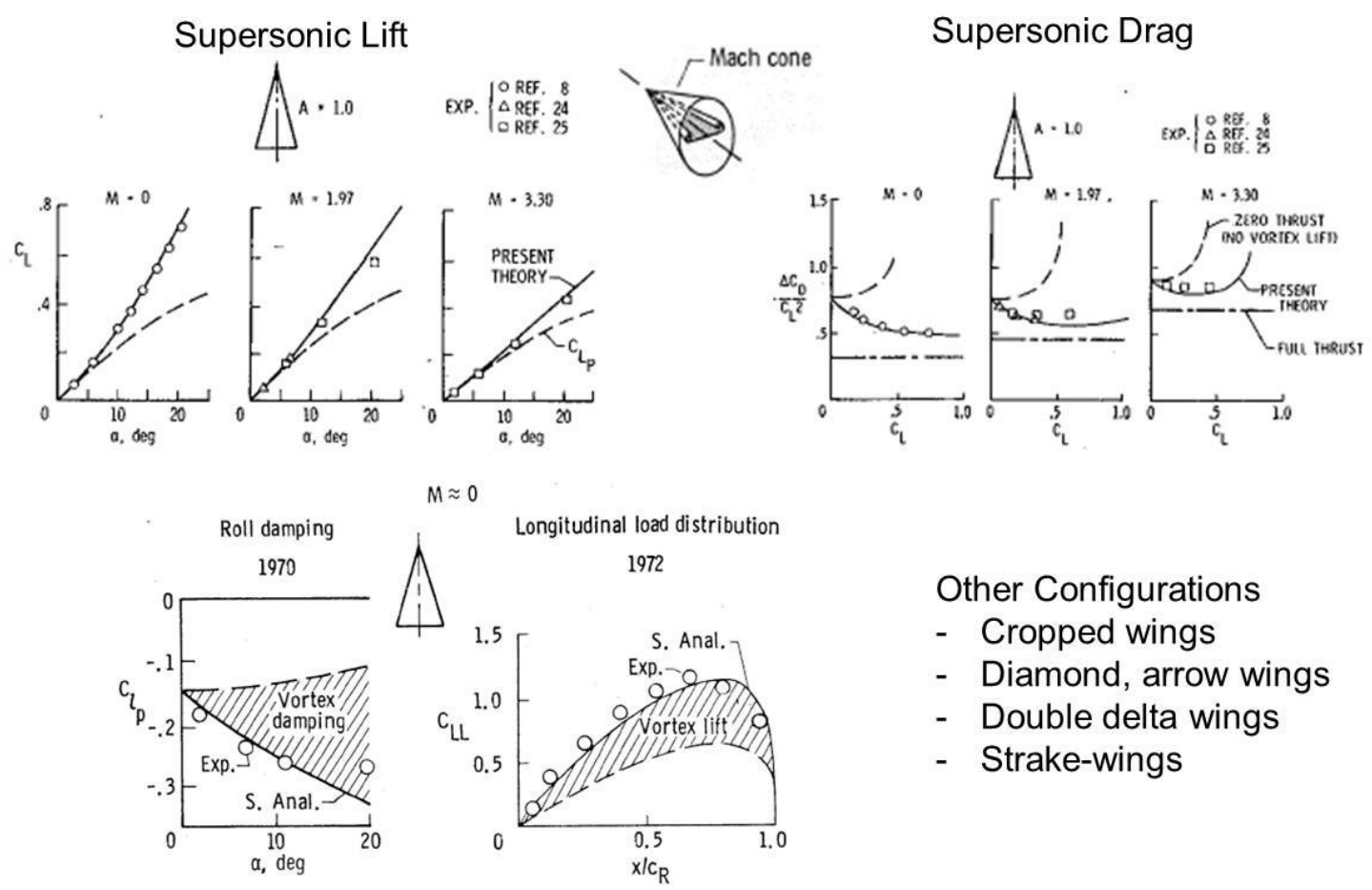

Figure 17. Suction analogy assessments.

Polhamus ${ }^{12}$ performed further delta wing assessments of the suction analogy for other aerodynamic parameters and other flow conditions, and some examples are shown in Figure 17. At supersonic speeds the vortex lift on a delta wing will diminish as the Mach cone approaches the wing leading edge. The results in the top left portion of this figure show a good estimate for this effect. The upper right portion of the figure has comparisons for the dragdue-to-lift parameter, $\Delta \mathrm{C}_{\mathrm{D}} / \mathrm{C}_{\mathrm{L}}{ }^{2}$, at a subsonic and two supersonic Mach numbers, and again shows good correlation. Part of Polhamus' branch performed dynamic stability research, and the lower left portion of the figure shows a comparison for the roll damping parameter, $\mathrm{C}_{\mathrm{l}_{\mathrm{p}}}$, with the additional roll damping that was realized from the antisymmetric leading-edge vortex effect. The lower portion of the figure also shows a longitudinal load distribution for a delta wing with reasonable correlation between the suction analogy and experiment. This implied that pitching moments could be estimated with the suction analogy, and later work confirmed this to be the case.

The early computations to support the suction analogy assessments were performed by John Lamar ${ }^{13}$ with a modified Multhopp code he had developed for performing configuration aerodynamic assessments in Polhamus' branch. Other techniques ${ }^{14}$ were also used in the pursuit of more general planform assessments, ${ }^{15}$ and a very successful teaming with another Branch at Langley led to the development of a vortex-lattice code with the suction analogy implemented. This vortex-lattice method, developed by Margason and Lamar ${ }^{16}$, allowed for increased planform generality and linearized compressibility effects; it was later extended for more general configuration capabilities by Lamar and Gloss. ${ }^{17}$ (Most of these codes were included on the timeline Figure 9.) Polhamus' focus was always on the aerodynamics, and these codes were tools that supported the aerodynamic research within his branch.

As a supervisor, Polhamus was focused on nurturing the professional growth of his staff, and others who worked with the branch, to contribute aerodynamic advancements on problems of national interest. Lamar had the concept for extending the leading-edge suction analogy to account for separation-induced vortices from sharp side edges. With Polhamus' encouragement, Lamar ${ }^{18}$ developed the theory, implemented it numerically, designed a family of wings that he tested in the 7- by 10-Foot High-Speed Tunnel ${ }^{19}$ to assess the theory, and published the results. Polhamus was also sponsoring the external development of more advanced vortex-flow methods for the prediction of three dimensional wing pressure ${ }^{20}$ and that could perhaps replace the suction analogy. This was the environment in Polhamus' branch. 
The initial suction analogy development spanned 1966 to 1971, and this time frame overlapped with the initiation of the Lightweight Fighter Program (LWF) in the United States. This program was a departure from more traditional development programs of that era in that the LWF aircraft was intended to be small, relatively inexpensive, and incorporate high-angle-of-attack maneuver capability. This program produced the General Dynamics F-16, and led to the later development of the McDonnell Douglas F18. The timing of the suction analogy development was fortunate, in that vortex-lift increments for slender wings with sharp edges could now be estimated with some confidence and with a relatively simple technique.

In the case of the F-16 development, many design objectives had been met with early concepts that featured a highly blended wing-body and a flattened forebody with blunt leading edges. See Figure 18 from $\mathrm{Smith}^{21}$ et al. Maneuver aerodynamics were however still lacking. Polhamus' branch was well connected with industry, and early consulting with Langley led to a design change recommendation to change the blunt-

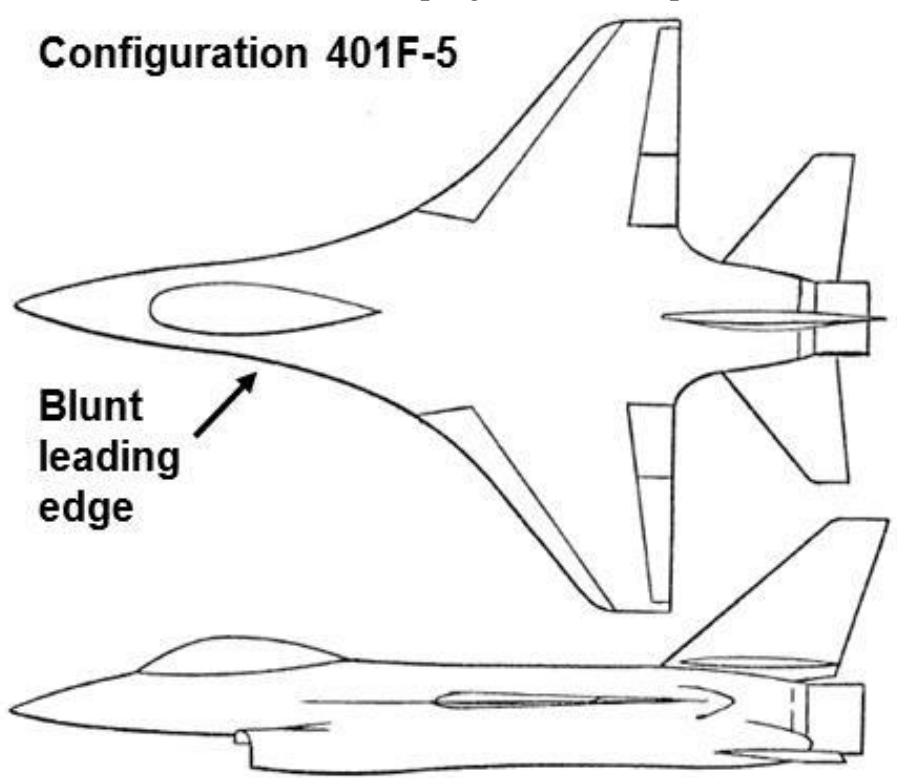

Figure 18. Early configuration concept with blended and blunt-leading-edge forebody, General Dynamics. Smith ${ }^{21}$ et al.

leading-edge forebody (an attached flow design) to a sharp-edged forebody (what became a strake) to take advantage of controlled vortex separation at high angle-of-attack maneuver conditions (see Smith ${ }^{21}$ et al.). The General Dynamics engineers proceeded to tailor the sharp-edged strake and wing-empennage combination to make the additional lift useable and solved many other practical challenges. All of this work was done experimentally, and their work resulted in the F-16. Subsequent research in Polhamus' branch led to the prediction and understanding of strake-wing aerodynamics with sharp-edged vortex flows. ${ }^{22}$ This work was accomplished after the development and initial flight of the F-16.

The fortunate timing of Polhamus' suction analogy was not restricted to the F-16. The concept of controlled separation and vortex lift had become wide spread, and many other independent developments of aircraft exploiting this design approach were realized. A chronological summary of aircraft that used vortex lift is shown in Figure 19 along with the time for the initial leading-edge suction analogy publications by Polhamus. It is not known if the suction analogy itself was directly used for the development of these vehicles, but it had become widely known and endorsed, and it seems as Polhamus' work could have contributed some confidence to the use of the sharp-edged vortex flows as more advanced methods were being developed and experimental experience was being accrued. 


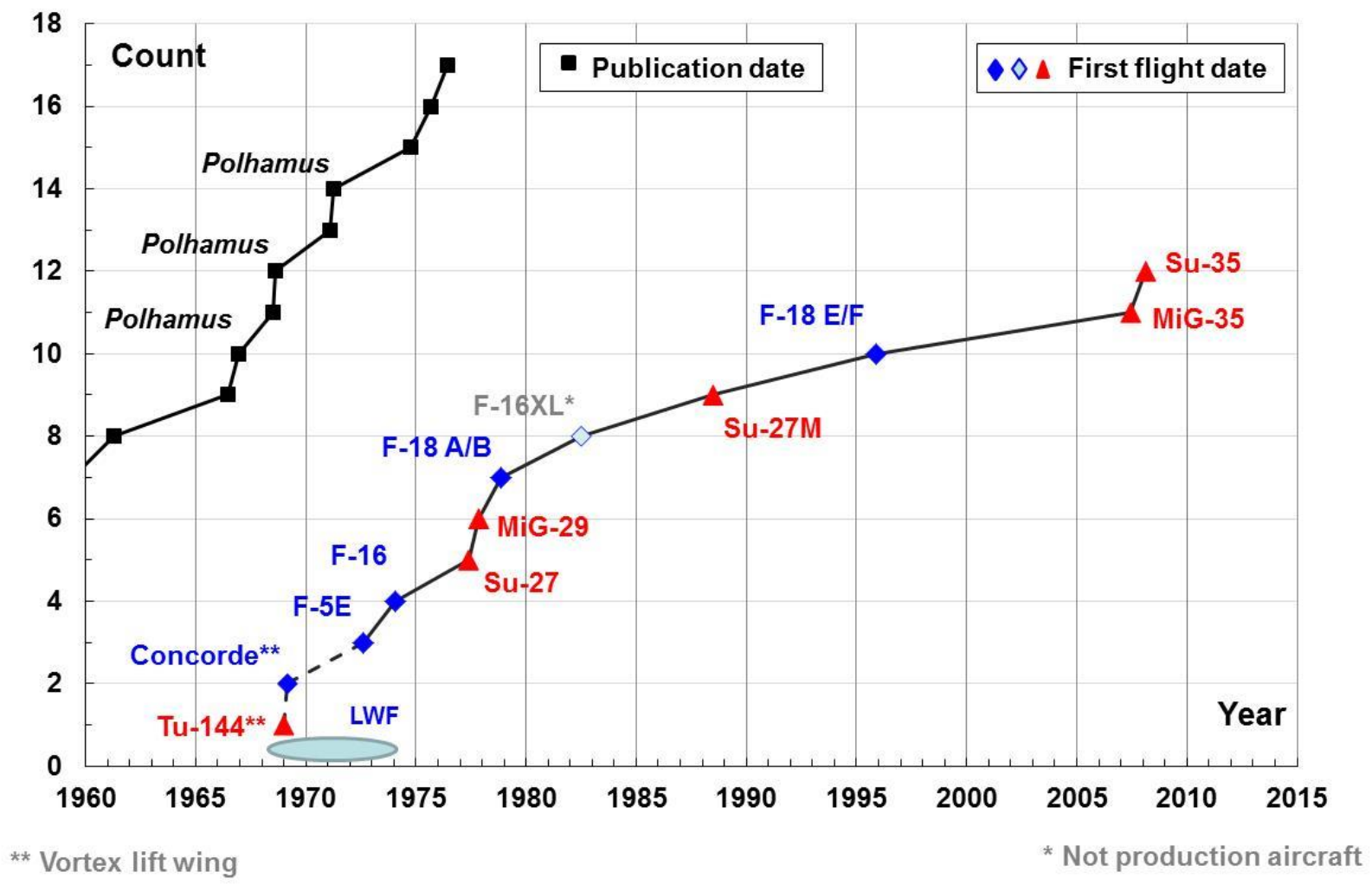

Figure 19. Vortex lift: prediction papers and aircraft. 


\section{Variable-Sweep Research}

Polhamus made a critical contribution to the realization of a practical variable-sweep capability for high-speed aircraft. This work predated his contributions to leading-edge vortex flows. A brief summary of this contribution is presented in this section. The background for this work again takes us back to the end of War II.

\section{A. Early Work}

The benefits of wing sweep had been discovered by Adolf Busemann ${ }^{23}$ in 1935 and later independently by R. T. Jones $^{24}$ in 1946. Toward the end of World War II, a prototype aircraft under development by Messerschmitt, the P1101 , was discovered by the allies that had provision for changing the wing sweep. This full-scale prototype was not intended for flight, and it could have the wing leading-edge sweep manually changed on the ground between $35^{\circ}$ and $45^{\circ}$. A sketch of this configuration is shown in part (a) of Figure 20. The P-1101 prototype was sent to the United States for study, and although damaged in transit, it became the baseline for the first aircraft capable of changing its wing sweep in flight, the Bell $\mathrm{X}-5$. The X-5 was a research aircraft, operated by the NACA, and it is shown in part (b) of Figure 20. (The small inset shows Laurence D. Bell.) The first flight of the Bell X-5 was in June of 1951, and the wing sweep could be changed in flight to $20^{\circ}, 40^{\circ}$, or $60^{\circ}$. It was known that sweeping the wings aft would cause a serious problem with the longitudinal shift in aerodynamic center, and, to compensate for this problem, the Bell X-5 wing pivot point, on the aircraft centerline, was shifted forward as the wings swept aft through a complicated jack-screw arrangement. The X-5 flight research program was successful despite various limitations that the research vehicle exhibited.

The concept of variable sweep was very attractive in the context of the slender wing aircraft being pursued for high speed flight. The slender wing planforms had poor low-speed behavior as mentioned in the preceding section, and this concept of being able to have a low-swept wing for low speed flight and a high-swept wing for high speed flight was one attractive alternative. Variable sweep also was gaining interest as a means to design a single airframe that could meet multiple mission requirements. However, the shift in longitudinal stability as wing sweep changed, and other practical concerns,

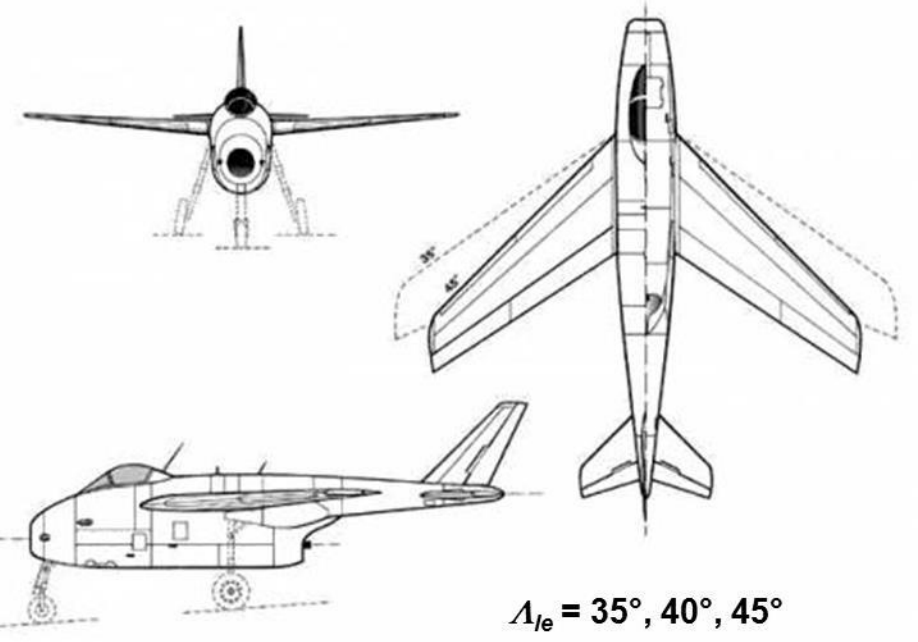

a) Messerschmitt P-1101.

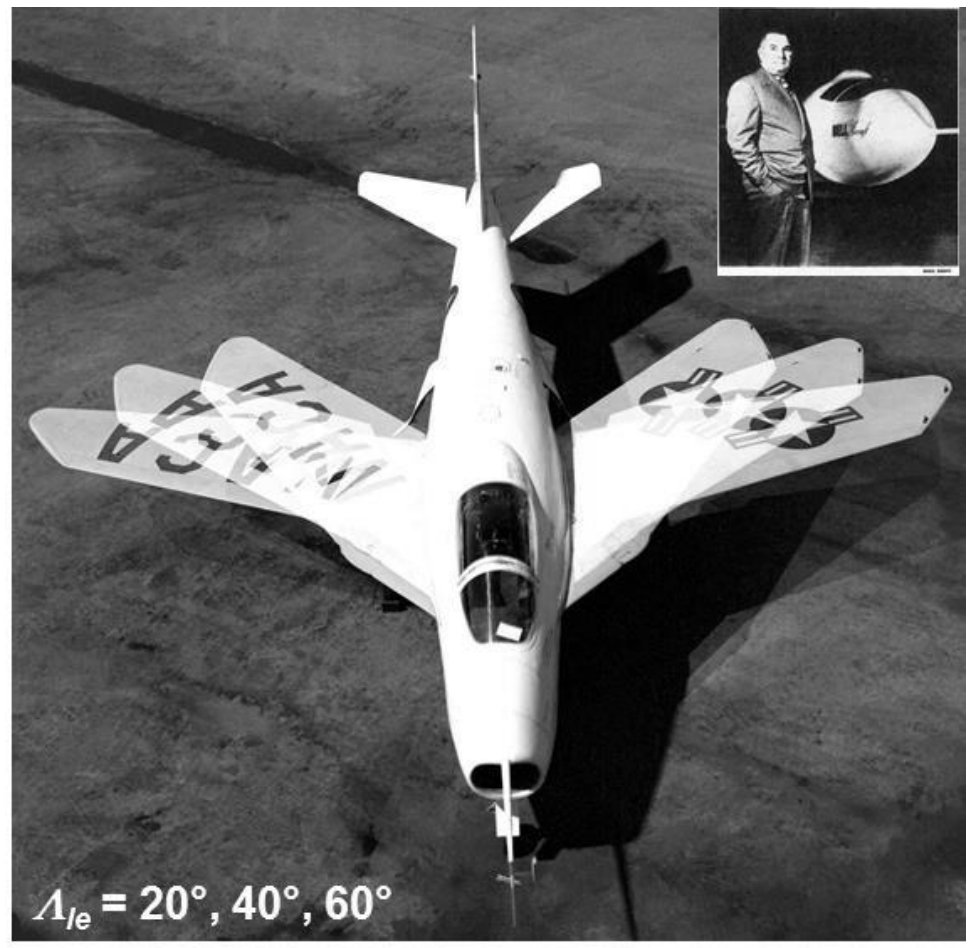

b) Bell X-5.

Figure 20. Early variable-sweep aircraft. would have to be resolved if the concept were to become a practical option for high-speed aircraft. 


\section{B. Focused Research}

Variable-sweep research had been underway at NACA Langley, initially in relation to the X-5 flight-test research program. A number of other programs established increased interest in the variable-sweep concept, and research accelerated during the 1950s in response. A brief summary of this research related to variable-sweep aircraft development has been given by Polhamus and Toll. ${ }^{25}$ Intensity of the variable-sweep research continued to grow, and in June of 1961 the country launched the first development program targeted at a variable-sweep aircraft, the Tactical Fighter Experimental (TFX) program. Throughout this timeframe the research continued to become increasingly focused toward issues that were critical for the successful creation of a new airframe incorporating variable sweep. Virtually all the wind tunnels at Langley were involved in the work, with each resident aerodynamics branch/tunnel contributing expertise to the many challenges that had to be addressed for variable sweep to become a practical option for high-speed aircraft. For configuration aerodynamics, these challenges included, but were not limited to, efficient transonic cruise, efficient supersonic dash, propulsion/airframe integration, stores integration, and stability and control characteristics.

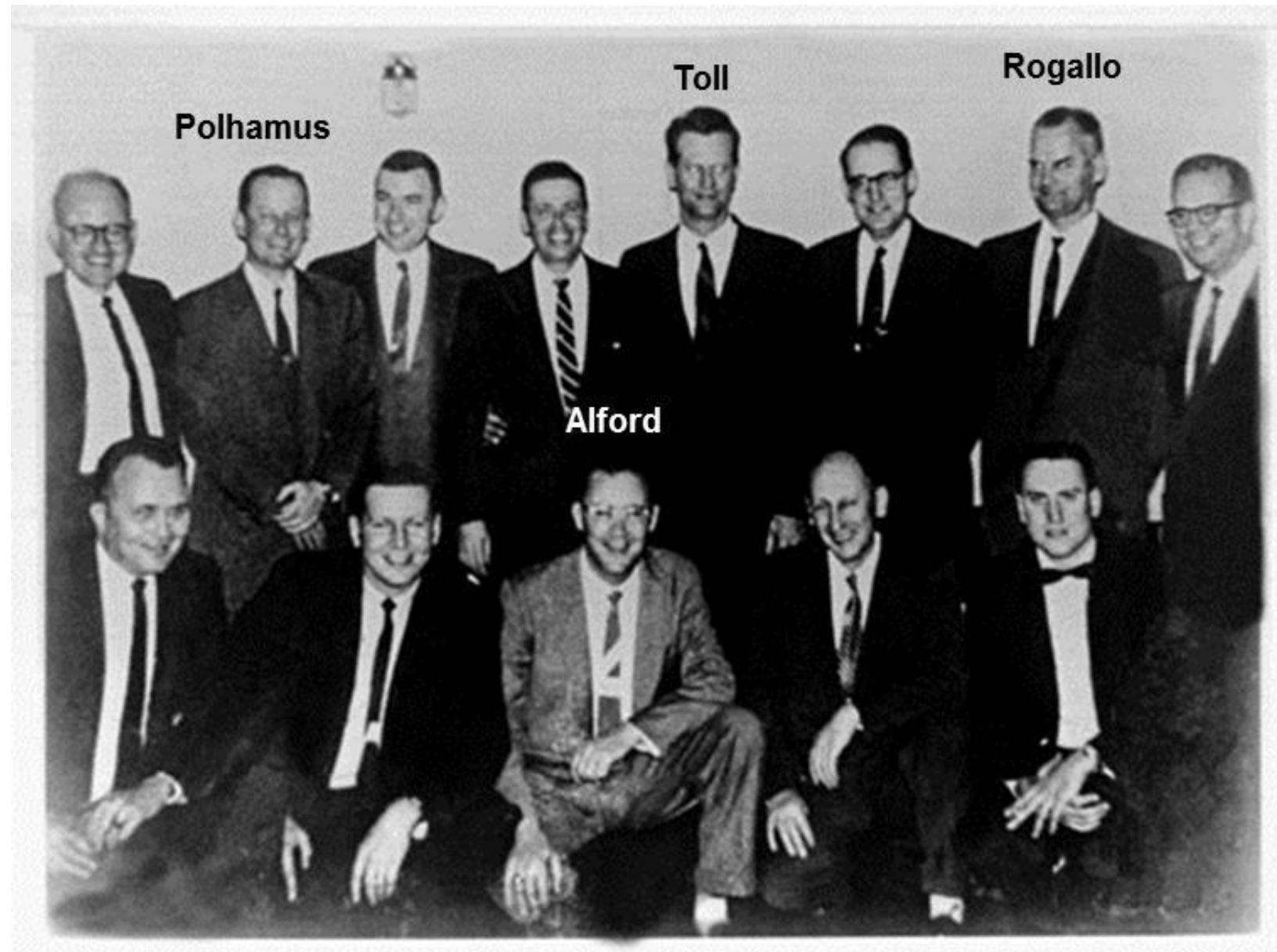

Figure 21. 7- by10-Foot High-Speed tunnel research staff, 1958.

Top row: William (Bill) C. Hayes, Edward (Eddie) C. Polhamus, William (Bill) Kemp, Paul G. Fournier, Thomas (Tommy) A. Toll, Richard (Dick) E. Kuhn, Francis (Rog) R. Rogallo, William (Bill) C. Sleeman

Bottom row: Robert (Bob) T. Taylor, Bernard (Buzzard) Spencer, William (Joe) J. Alford, Kenneth (Ken) P. Spreeman, Wilson Thompson

Much of the basic stability and control work was performed in the 7- by 10-Foot High-Speed Tunnel where Polhamus worked. The research branch had extensive background in configuration aerodynamics for many aircraft concepts at subsonic compressible speeds, and the 7- by 10-Foot High-Speed Tunnel was a very productive and flexible facility at Langely. The 7- by 10-Foot High-Speed Tunnel research staff is shown in Figure 21 at the Chamberlin hotel, Hampton Virginia, in 1958 for the commemoration of Tommy Toll's selection to become the 
Deputy Director for Flight Research at the NASA Dryden Research Center. Also noted in the figure is Francis Rogallo, who would develop the para-wing (this led to hang gliders), and Joe Alford who shared with Eddie Polhamus some breakthrough work on variable sweep.

The barrier problem that Polhamus worked from the 7- by 10-Foot High-Speed Tunnel branch was the unacceptably large shift in aerodynamic center as the wings were swept back. This problem is illustrated in Figure 22. In this example, the wing pivots about a fixed point on the vehicle centerline, and, as a consequence, the lift shifts aft with sweep angle. Although the center of gravity is also shifting aft with wing sweep, the center of lift is shifting aft at a greater rate, and the aircraft becomes too stable at the high sweep conditions. The wings are being swept back for high-speed flight, and compressibility exacerbates the excess stability. This problem not only resulted in unacceptable handling properties, but also unacceptable trim drag increments for high-speed flight.

Two options to solve this problem are shown in Figure 23. The top portion of the figure repeats the variable-sweep wing sketch with an inboard (centerline) fixed pivot point. The middle sketch shows the variable-sweep wing with an inboard translating pivot point. This option was used with the Bell X-5 research aircraft, but was impractical for production aircraft due to mechanical complexity and weight among other factors. The bottom sketch shows the variable-sweep wing with a fixed outboard pivot point. With the fixed outboard pivot point, the variable-sweep wing could approximately match the wing locations achieved with the inboard translating pivot point, but with greatly reduced mechanical complexity. This was a promising concept.

The fixed outboard pivot approach meshed well with a gloved-wing concept, and a sketch of a gloved-wing with variable sweep is shown in Figure 24. This approach to variable sweep with an outboard pivot point was assessed experimentally, and the results demonstrated that the shift in aerodynamic center with wing sweep had been greatly reduced as shown in the sketch on the right side of Figure 24. Details of the pivot location, the glove geometry, the

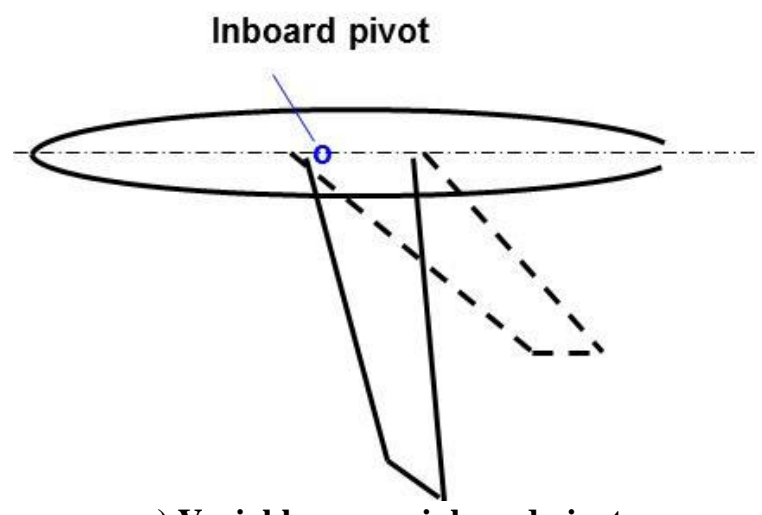

a) Variable sweep, inboard pivot.

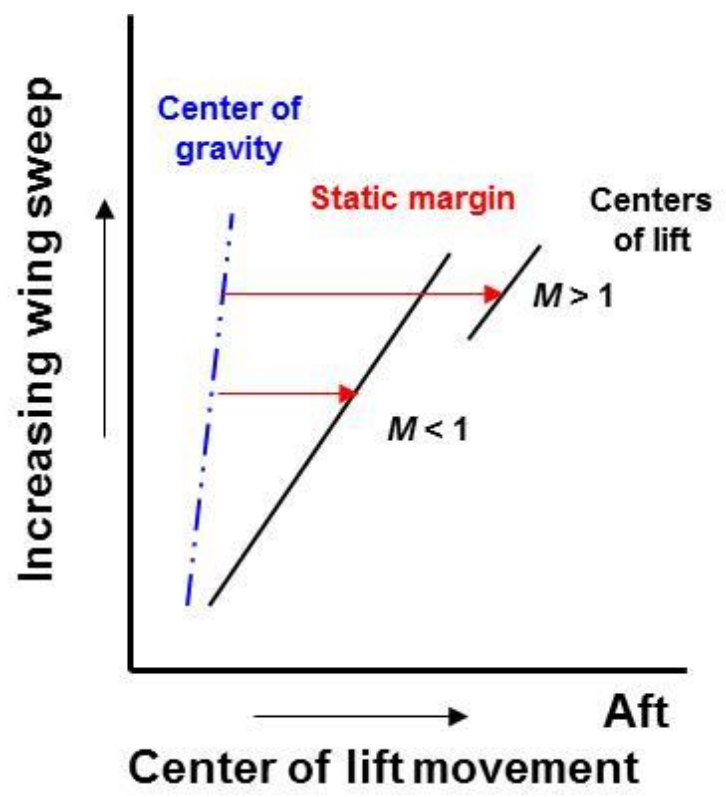

b) Change in stability with sweep.

Figure 22. Stability problem, variable sweep with inboard pivot.

wing-body interface, and so forth were important to the many other aerodynamic challenges in designing an aircraft with variable sweep, and these challenges could now be addressed with this new approach. 


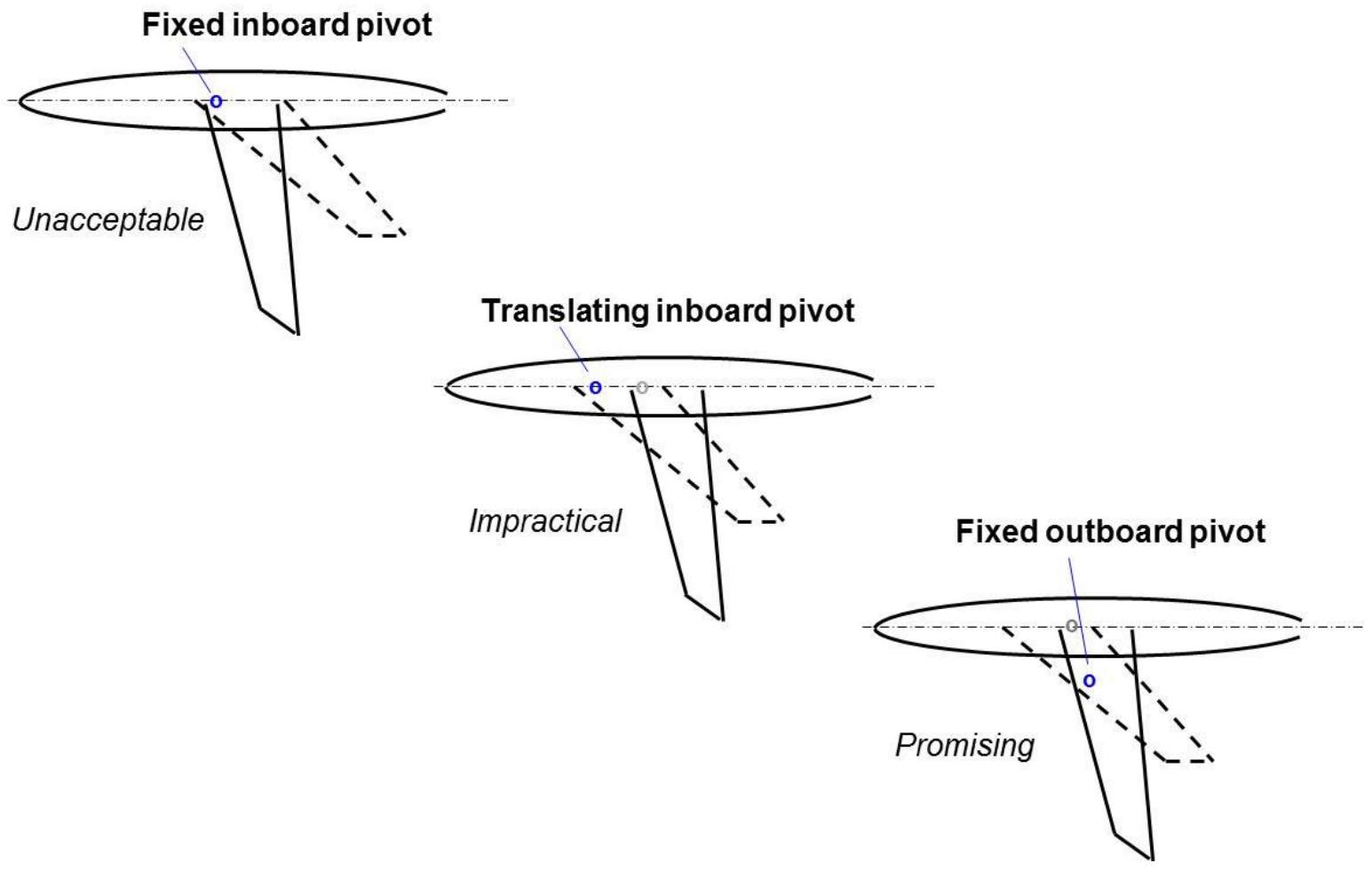

Figure 23. Concepts to reduce aerodynamic center shift, variable sweep.
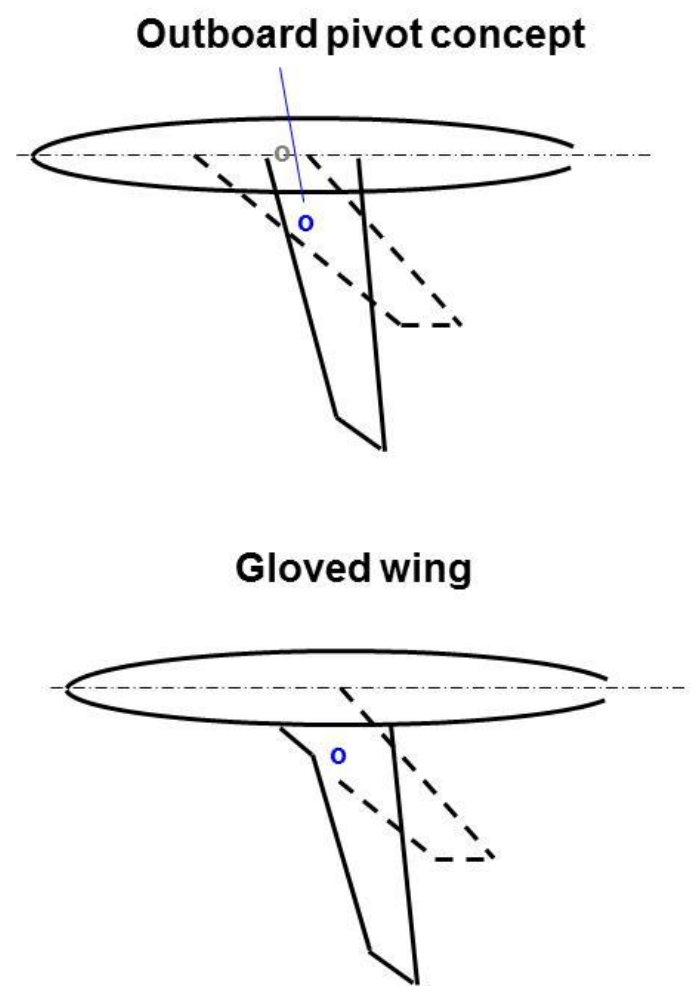

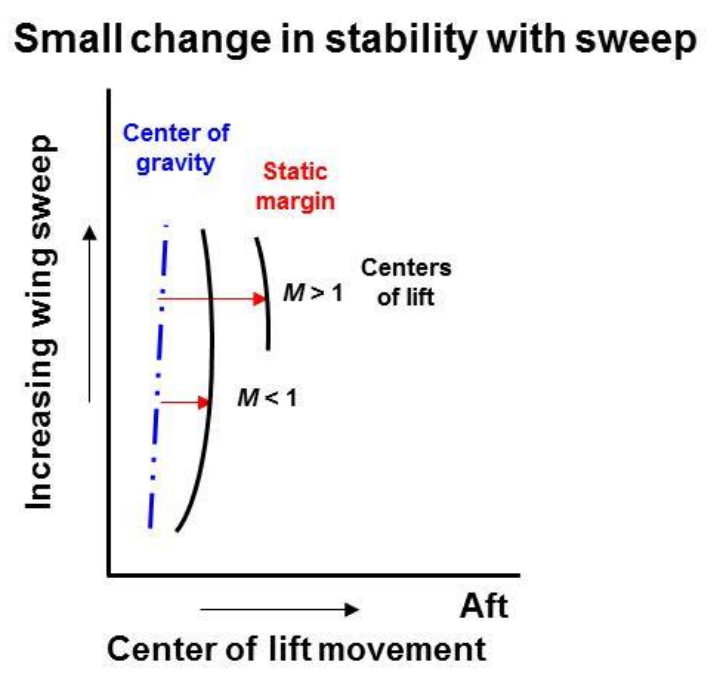

Figure 24. Outboard pivot concept, variable sweep. 


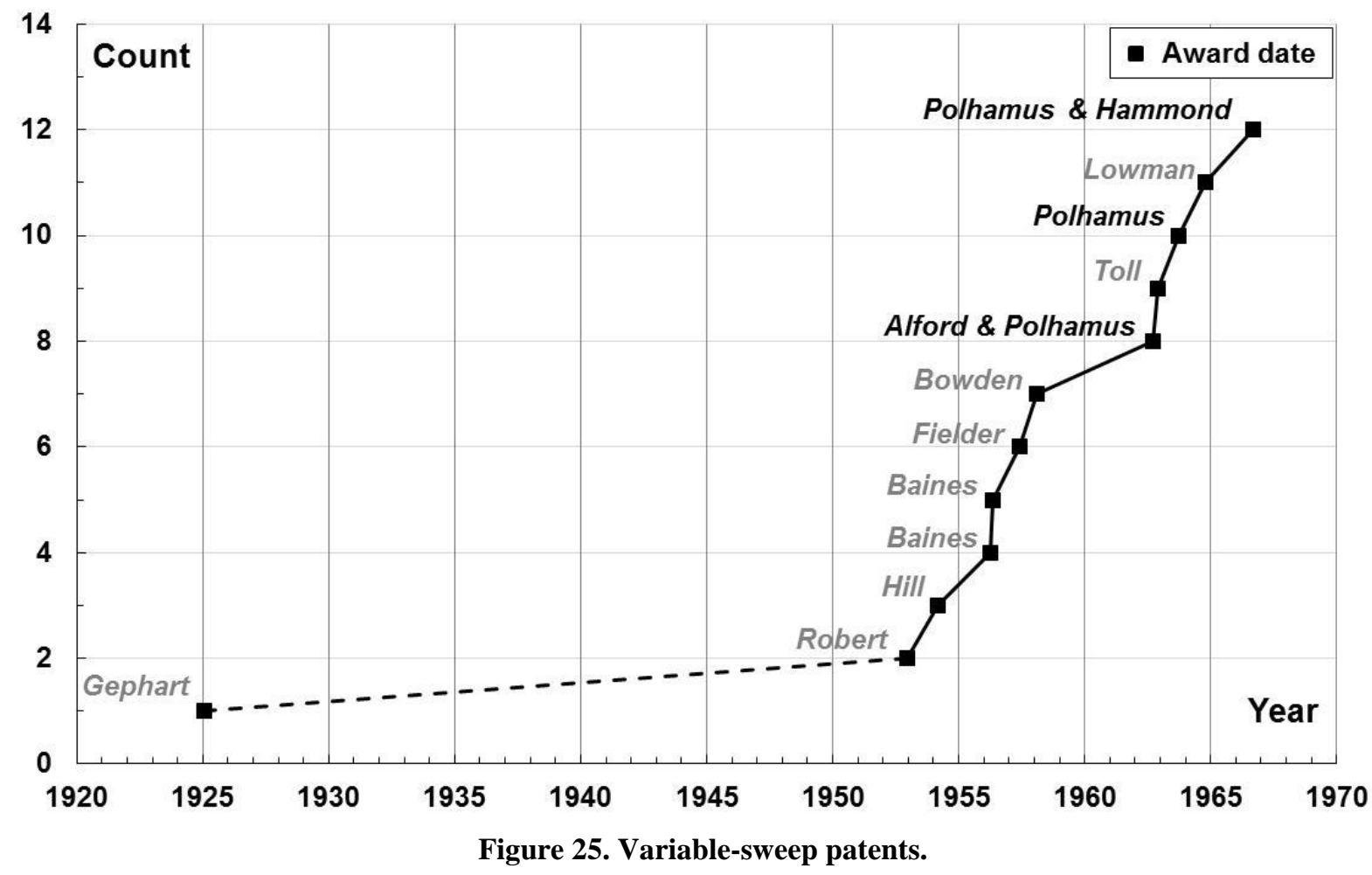

Polhamus was awarded three patents ${ }^{26,27,28}$ for the variable-sweep concept with an outboard pivot. A timeline of the award dates for these three patents is shown in Figure 25 along with the relevant prior patents of related concepts. New concepts must be sufficiently unique to earn a patent, and the awarded patents on the figure reflect the aggressive research that was underway in the 1950s on variable sweep. However, it was surprising to find a challenge to Polhamus' new work on variable sweep with an outboard pivot point from a patent awarded in 1925. In the early 1920s a gentleman by the name of Valentine Gephart had been interested in storing airplanes in small spaces, such as a garage, and he devised a wing pivoting mechanism that allowed the wings to be folded alongside the fuselage for this purpose. Gephart ${ }^{29}$ had the foresight to patent this idea, and the drawing from his patent of his airplane, with folding wings for storage, is shown in Figure 26. Gephart's initial drawings include a front view of his airplane with the wings folded along the fuselage. It was not too difficult for Polhamus to distinguish his work on inflight variable sweep, with an outboard pivot, from Gephart's work, and the corresponding initial drawings from Polhamus' three patents are shown in Figure 27.

With the success of the outboard pivot, variable sweep became a practical option for aircraft. Many other challenges were also addressed, and as a consequence airframe companies could realistically assess variable sweep as an approach to meet the multi-mission interests of national programs from that era. The evolution from a research aircraft to a deployed airframe is summarized in Figure 28. The left portion of the figure shows the NACA Bell X-5 research aircraft that flew between June of 1951 and December of 1958. During the 1550s and 1960s, research was underway in the wind tunnels of Langley to address the many challenges associated with the variable-sweep concept, and the configuration shown in the middle of Figure 28 is one of the Combat Air Patrol (CAP) wind tunnel models that were tested in support of naval interests. The right portion of the figure shows the first variable-sweep aircraft, the F-111, with the wings swept near the two extreme values that were possible.

A partial timeline of the variable-sweep patents shown in Figure 25 is repeated in Figure 29 along with the date of first flight for aircraft that used variable sweep. The TFX program is also indicated, and the outcome from this program was the F-111. Ten of the twelve aircraft shown became production aircraft, and every one of these variable-sweep airplanes used the outboard pivot concept developed by Polhamus. (The AD-1 research aircraft has been omitted from this chart only because the purpose of the oblique wing was so different from the other highspeed aircraft shown.) 
AIAA $34^{\text {th }}$ Applied Aerodynamics Conference Washington, DC

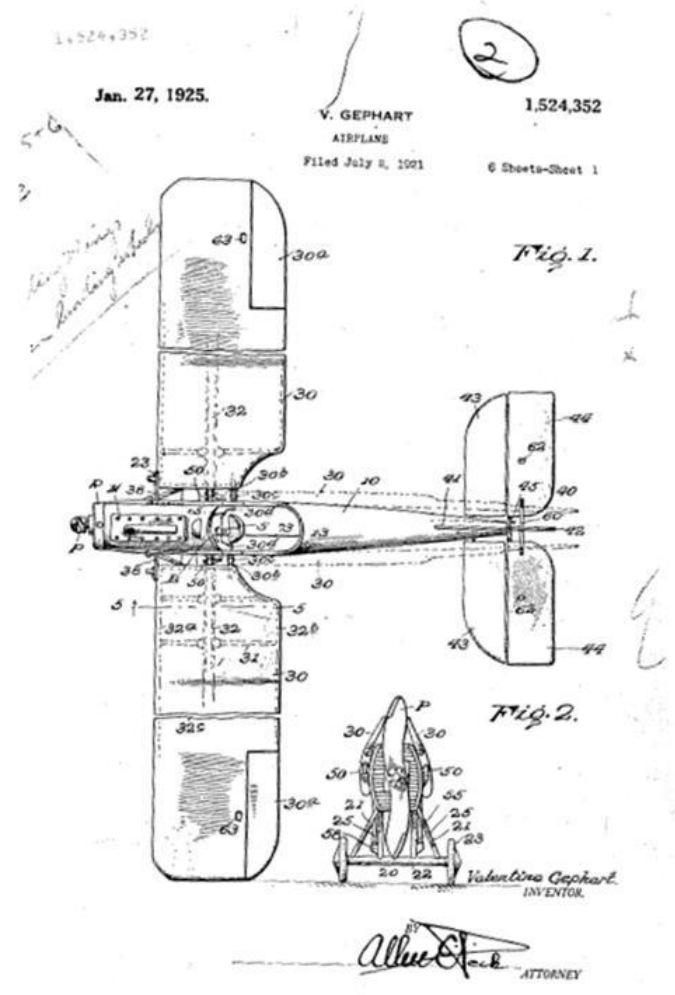

AIAA 2016-xxxx

Historically Significant Papers

Figure 26. United States patent 1,524,352 awarded to Valentine Gephart, January 1925.

\section{Alford \& Polhamus}

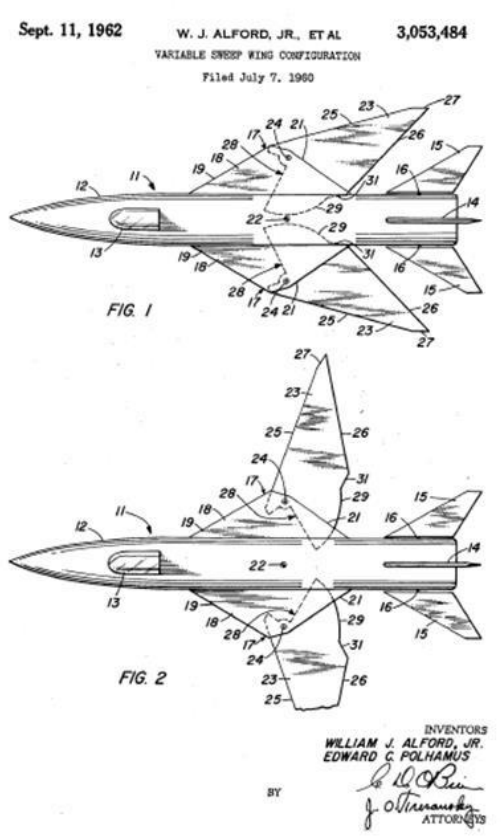

Polhamus
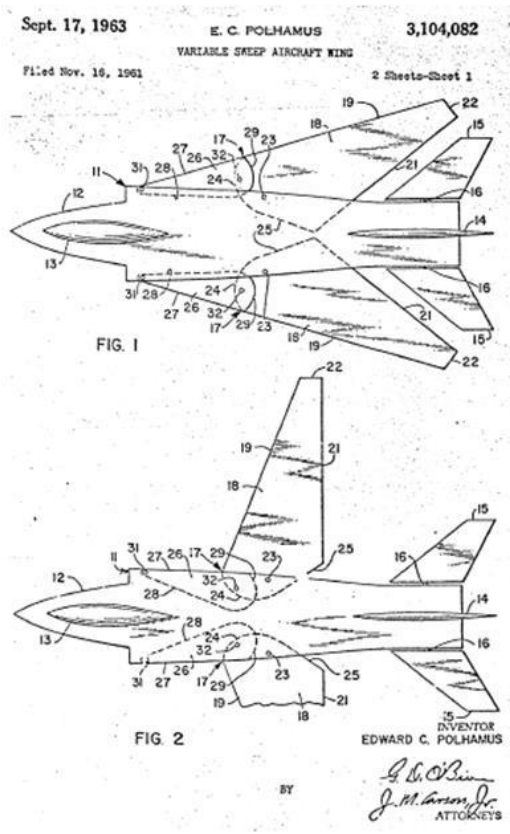

Polhamus \& Hammond

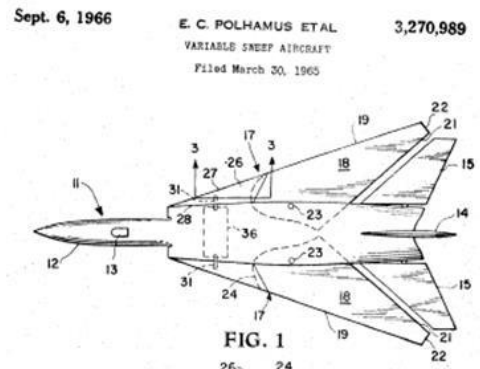

FIG. 3
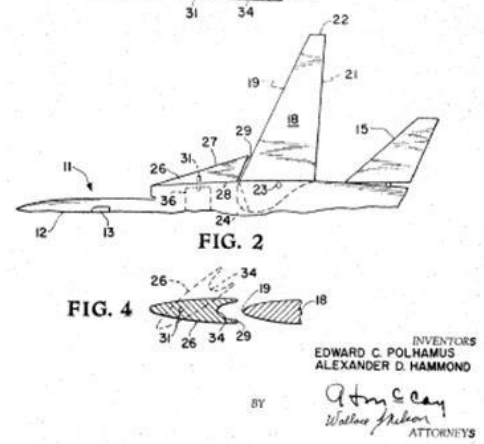

Figure 27. United States patents for variable sweep with an outboard pivot. 

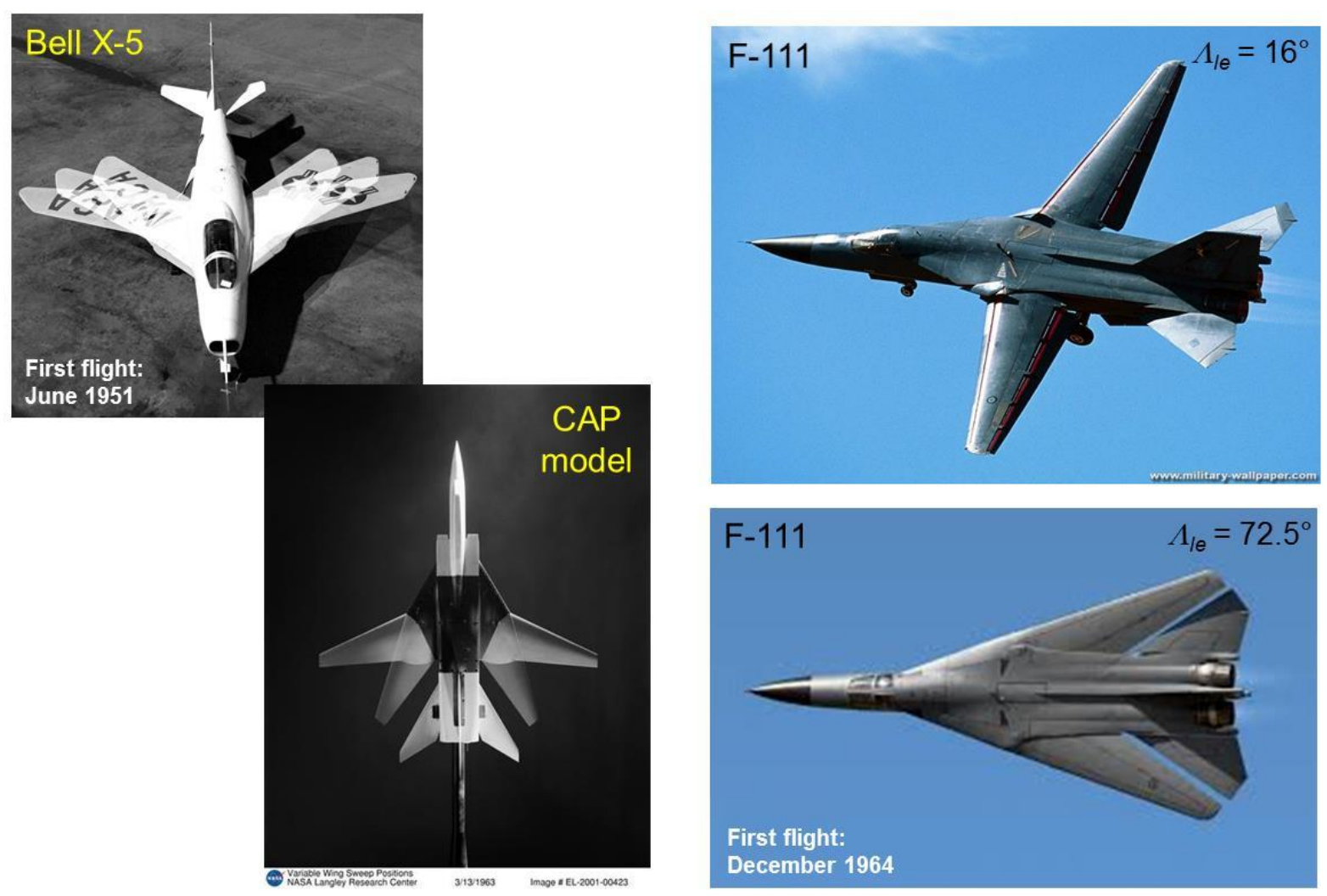

Figure 28. Evolution of variable sweep.

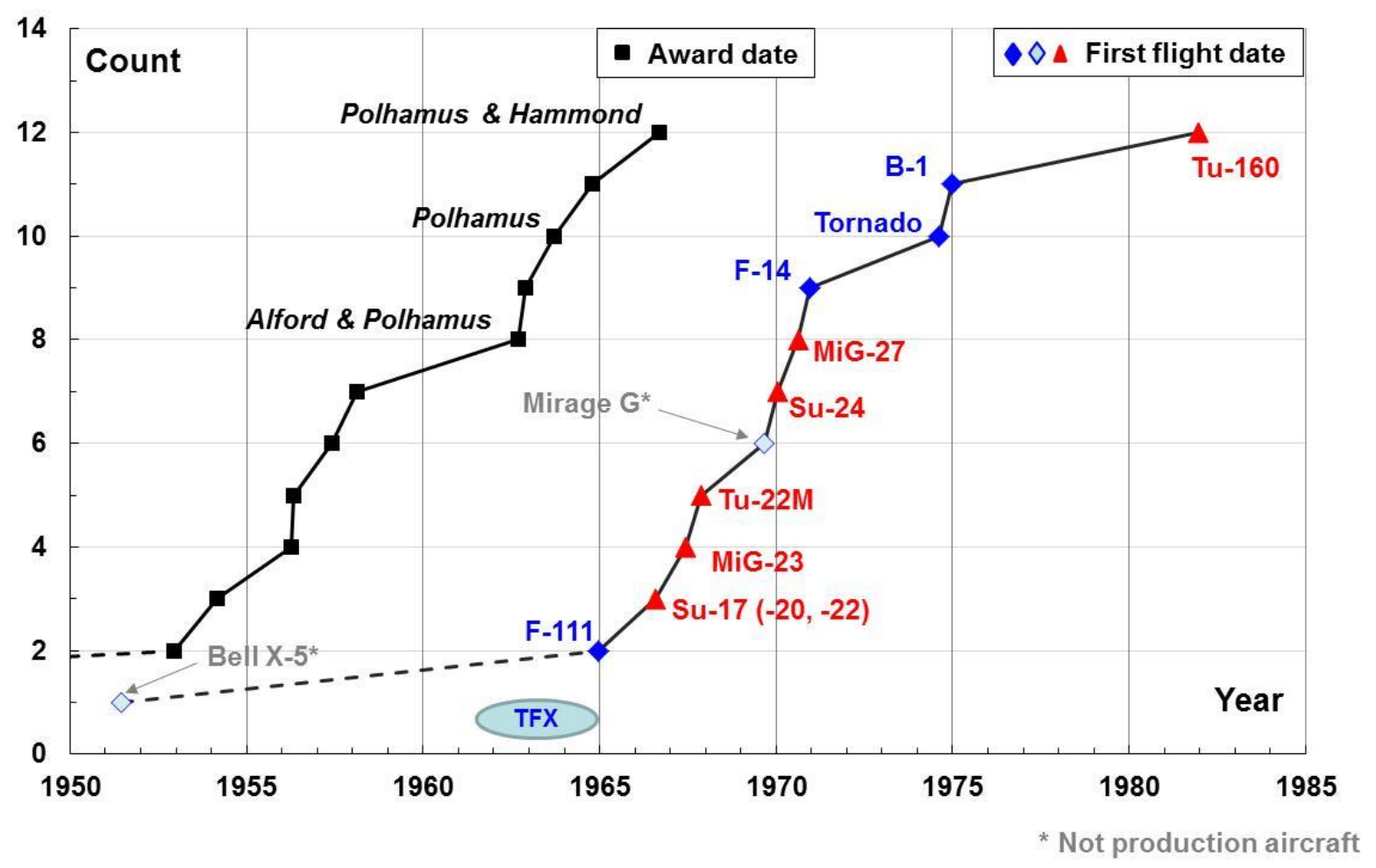

Figure 29. Variable-sweep patents and aircraft. 


\section{Reynold Number Effects Research}

Polhamus also made critical contributions to wind tunnel test capabilities to obtain Reynolds number effects and high Reynolds number data through a cryogenic concept. This topic will only be touched on briefly.

\section{A. Background}

Polhamus' interest in Reynolds number effects appear to go back to the beginning of his career, when the DM-1 tests, discussed earlier in this report, were performed at Langley. The full-scale DM-1 vehicle could be tested at low speeds in the 30- by 60-Foot Tunnel at Langley, and then subscale model testing provided some insights to the Reynolds number effects of that unusual configuration. Of course, most aerodynamic testing can only be performed at subscale Reynolds numbers and the aerodynamics at full-scale Reynolds number conditions are left to be determined by extrapolation techniques. Pressure tunnels have helped with this problem, but are still limited in many applications to subscale conditions.

Cryogenic temperatures cause significant reductions in kinematic viscosity, and thus provide an alternate means to pressurization for creating a high Reynolds number flow. This basic cryogenic concept for high Reynolds number flows had been discussed by Margoulis ${ }^{30}$ in 1920 and by Smelt ${ }^{31}$ in 1945, but it had not been implemented for aerodynamic assessments in a transonic wind tunnel.

\section{B. Transonic Cryogenic Tunnel}

Polhamus established a small group within his branch in 1968 to begin exploring the practicality of a cryogenic wind tunnel with transonic capability. Conceptual studies were performed in a first phase. An attractive aspect of cryogenic high Reynolds number testing is shown in Figure 30 from Polhamus $^{32}$ et al. The results in this figure are for a fixed Mach number, and they illustrate the very high Reynolds numbers that can be achieved at cryogenic conditions (factor of 5 compared to an ambient temperature). The thermodynamic variations in density and velocity (at fixed Mach number) result in a constant dynamic pressure, and, hence, constant model loads. Because the speed of sound is diminishing with temperature, the drive horsepower to sustain the flow diminishes as temperature is decreased. However, to realize these capabilities, many fundamental questions would need to be addressed from both a gas dynamics and a facility operations perspective. This first phase also included early experimental

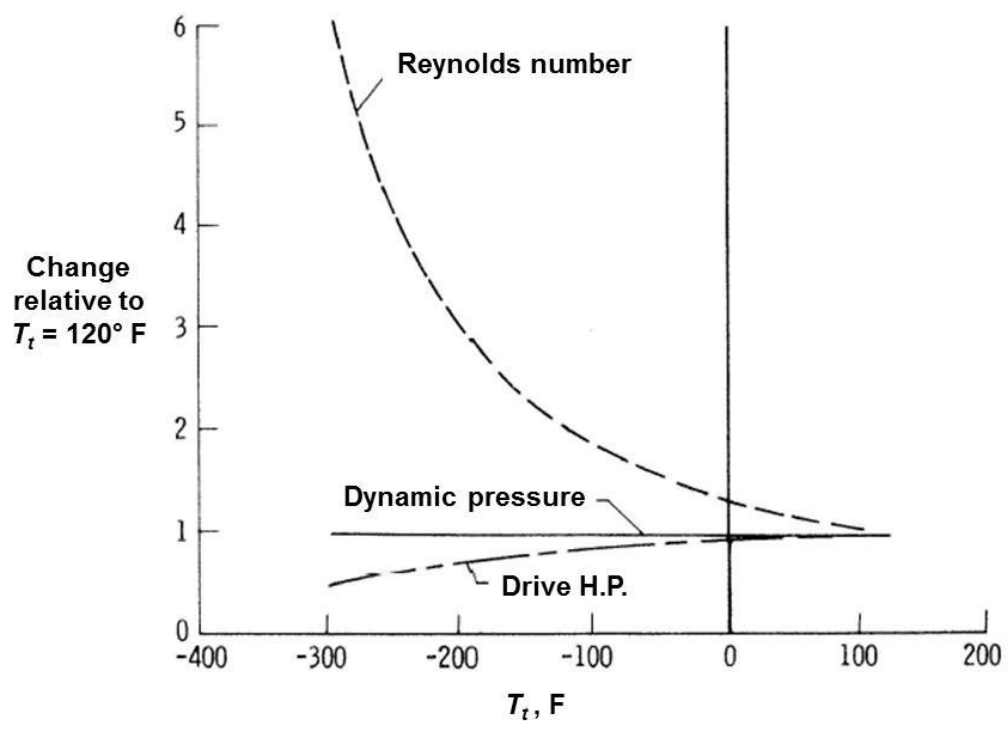

Figure 30. A key argument for cryogenic wind tunnels, $\mathrm{M}=$ const. From Polhamus ${ }^{32}$ et al. assessments in a small low-speed, closed circuit tunnel (fabricated from plywood) that used cryogenic nitrogen as the test medium.

Enough progress was made from the early conceptual and experimental work to submit a proposal in February of 1971 for the design and fabrication of a pilot cryogenic wind tunnel with transonic capability. The proposal was accepted, work began, and the 0.3-m Transonic Cryogenic Tunnel (TCT) became operational in September of 1973. A portion of the Branch research team involved in creating the 0.3-m TCT is shown in Figure 31, and many other people had become involved by this time. Polhamus had engendered a very effective team, and among the key contributions was the conceptual work from Kilgore ${ }^{33}$, developmental work from Goodyer and Kilgore ${ }^{34}$, real gas effects from Adcock ${ }^{35}$, and condensation work from $\mathrm{Hall}^{36}$.

The work with the $0.3-\mathrm{m}$ TCT could be considered a second phase in assessing cryogenics for transonic wind tunnel testing. The facility design allowed for independent control of pressure up to approximately 5 atmospheres, total temperature down to cryogenic conditions, and velocity into the transonic regime. This meant that independent control of Mach number, Reynolds number, and dynamic pressure could be achieved. Many practical operational 
issues were solved with the work in the $0.3-\mathrm{m} \mathrm{TCT}$, and the ability to perform transonic experimental aerodynamics, including high Reynolds numbers, was demonstrated.

Two examples ${ }^{32}$ from the $0.3-\mathrm{m}$ TCT work are included. The first example shows chordwise pressure distributions for an airfoil model tested in the 0.3-m TCT, Figure 32. Data are presented for a subcritical and a supercritical Mach number condition. For each Mach number, there are two data sets at the same Reynolds number, one that was achieved by increasing total pressure and the other that was achieved by decreasing total temperature. The measurements are identical, and demonstrate that the same flow was achieved. The results include transonic shock/boundary-layer interaction flow physics at a chord Reynolds number of 8.5 million.

The second example illustrates the expanded test capability in a cryogenic pressure tunnel, Figure 33 . The envelope was scaled for capability that could be realized in a larger cryogenic facility. The three degrees of freedom in operating conditions (total pressure, total temperature, speed) can be exploited to isolate aerodynamic effects. In the example shown, the Mach number is constant, and test conditions can be controlled to vary Reynolds number at a fixed dynamic pressure, or to vary dynamic pressure at a fixed Reynolds number. This allows for the isolation of Reynolds number and aeroelastic effects. These effects would be confounded if Reynolds number and dynamic pressure were being varied simultaneously (as in a pressure tunnel). The cryogenic pressure tunnel allows for the determination of Reynolds number effects as well as for obtaining high Reynolds number data. Other isolations can be accomplished (e.g., separation of Reynolds number and Mach number effects at fixed dynamic pressure).

Based on the accomplishments from the 0.3-m Transonic Cryogenic Tunnel, a proposal to build a large cryogenic transonic wind tunnel was accepted in 1975, and this tunnel became the National Transonic Facility (NTF). Some summary observations toward this next generation of cryogenic tunnels were published by Polhamu ${ }^{37}$ near the time of his retirement.

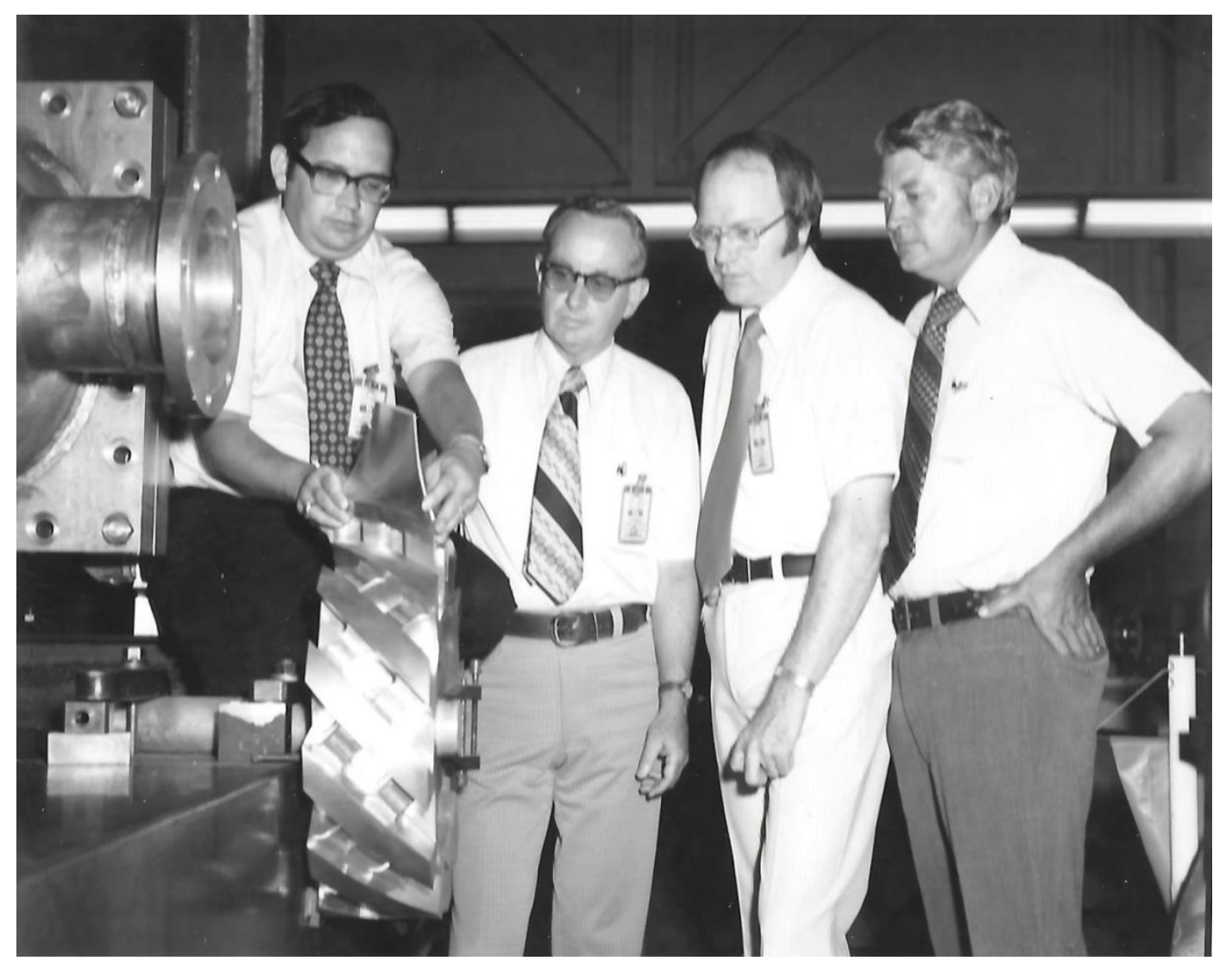

Figure 31. Part of the Branch research team that pioneered the creation of the 0.3-meter Transonic Cryogenic Tunnel, 1974. Left to Right: Jerry Adcock, Eddie Polhamus, Bob Kilgore, Ed Ray. 


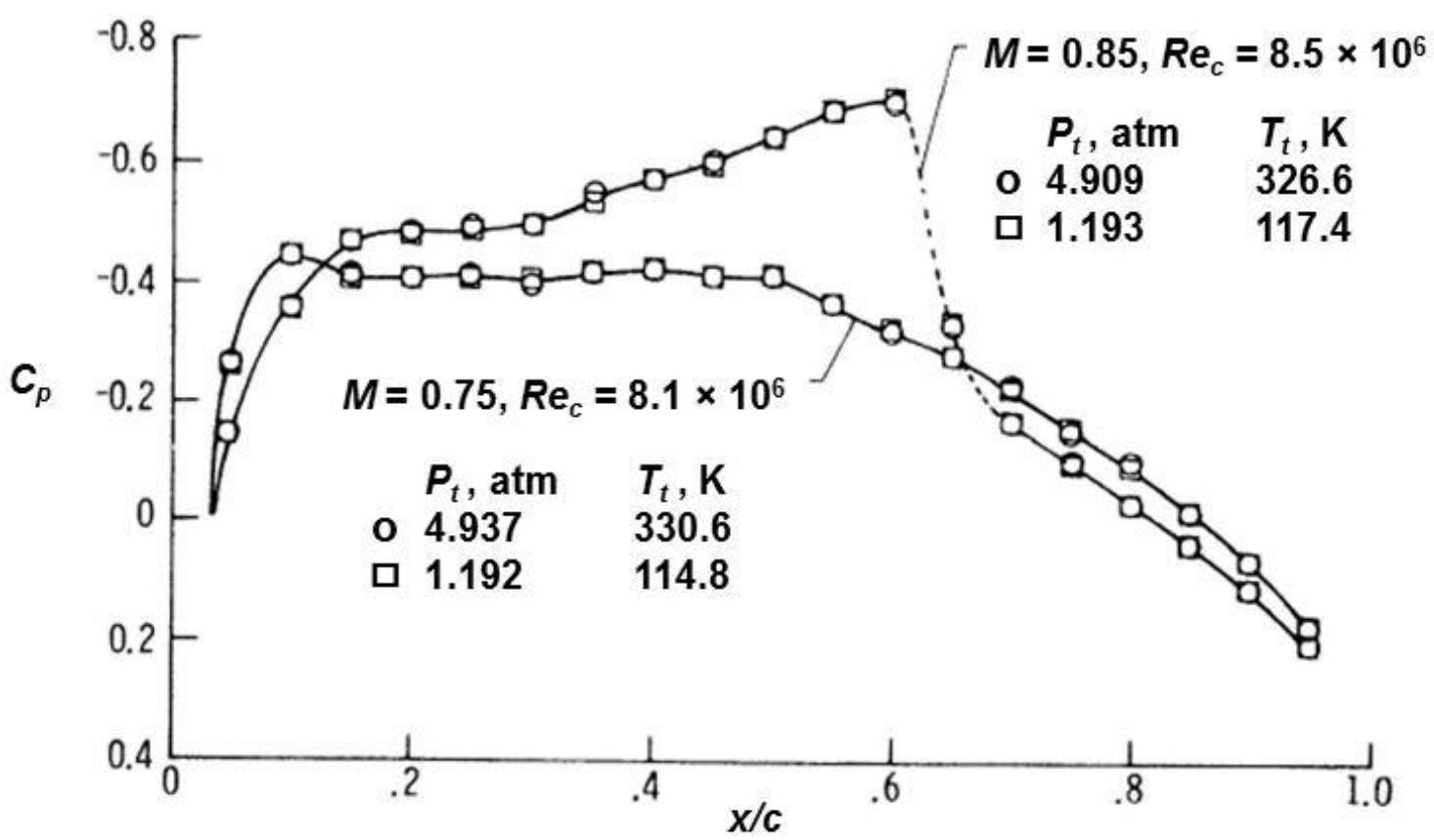

Figure 32. Airfoil pressure comparison. From Polhamus ${ }^{32}$ et al.

$M=1.0,8.2 \mathrm{ft} . \times 8.2 \mathrm{ft}$. test section

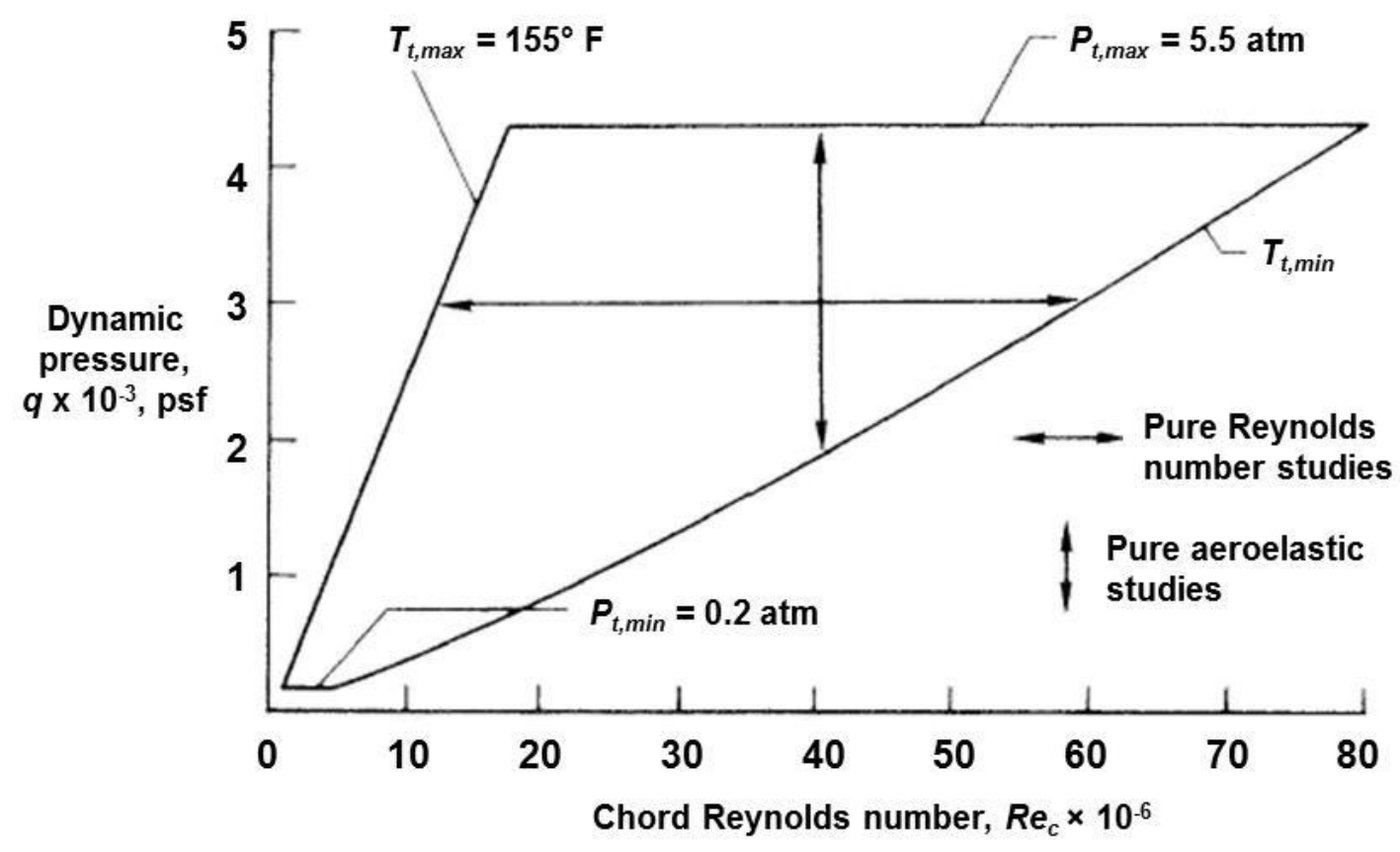

Figure 33. Example for isolation of Reynolds number and aeroelastic effects at constant Mach number. From Polhamus ${ }^{32}$ et al. 


\section{Recognition and Concluding Remarks}

Edward C. Polhamus had a very rich career at the NACA/NASA Langley research center. He was a humble individual, and, as mentioned earlier, he was more interested in the advancement and recognition of his employees and coworkers than himself. It is worth recapping some of the impacts his career has had from the topics covered in this paper:

- His development of the outboard pivot enabled the practical application of variable sweep for combat aircraft design.

- His development of the suction analogy provided the first accurate vortex lift predictions for wing aerodynamics.

- His leadership of the 0.3-m TCT work demonstrated the feasibility of the cryogenic concept for transonic wind tunnel testing.

There are further technical contributions from his career at NACA/NASA Langley, and he had a profound impact on the careers of many individuals who had the opportunity to work with him.

Despite Polhamus' understated nature, he was unable to avoid recognition for his work. In 1974 he received the NASA Medal for Exceptional Scientific Achievement "for outstanding leadership and personal contributions in developing aerodynamic technology that has significantly improved U.S. fighter aircraft and for leadership in developing advanced aerodynamic testing techniques and technology." He received a second NASA Medal for Exceptional Scientific Achievement in 1984 "for exceptional scientific achievement in leading the development of the cryogenic concept which led to acceptance of the concept for the National Transonic

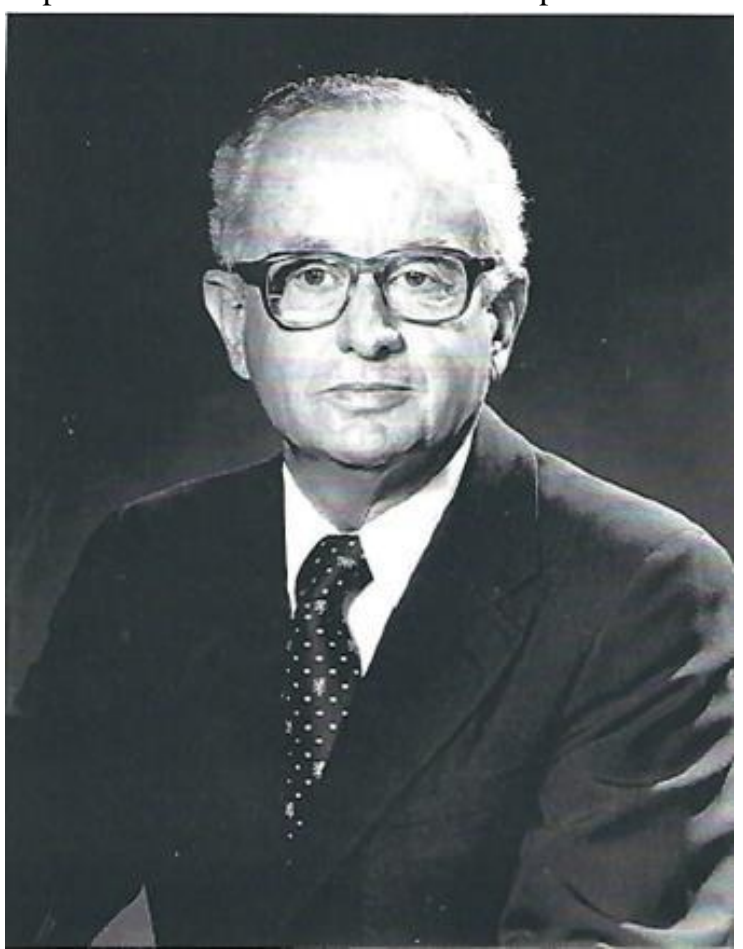

Figure 34. Edward C. Polhamus. Facility."

Polhamus also dedicated time to serve the aeronautical community for national and international interests. He was selected to serve on National Academy of Sciences Advisory Committees, was selected to serve on the AGARD Fluid Dynamics Panel for NATO, and was also selected to be the technical evaluator for the AGARD High Angle of Attack Aerodynamics symposium.

Polhamus was also recognized in the National Air and Space Museum's Flight Technology Gallery. At the time of this recognition, he was one of only six individuals to be so recognized. He was an Associate Fellow in the AIAA, and was selected to present the 1983 AIAA Wright Brother Lecture, ${ }^{38}$ "Applying Slender Wing Benefits to Military Aircraft.” A photograph of Polhamus near the completion of his career is shown in Figure 34.

\section{Acknowledgments}

This paper was invited by the Applied Aerodynamics Technical Committee for inclusion in their series on "Historically Significant Papers in Applied Aerodynamics," and this author is very appreciative of the opportunity to chronicle some of Edward C. Polhamus' aerodynamic contributions. The work was supported by the Transformative Aeronautics Concepts (TAC) Program, Transformational Tools and Technologies (TTT) Project, and this support is gratefully appreciated. The author also wishes to thank many associates from industry, academia and NASA Langley who helped with this material.

\section{References}

${ }^{1}$ Polhamus, E. C., "Predictions of Vortex-Lift Characteristics by a Leading-Edge Suction Analogy," AIAA Journal of Aircraft, Vol. 8, No. 4, 1971.

${ }^{2}$ Wilson, H. A., and Lovell, J. C., "Full-Scale Investigation of the Maximum Lift Flow Characteristics of an Airplane Having Approximately Triangular Planform,” NACA RM L6K20, Feb. 1947. 
${ }^{3}$ Chambers, J. R., "Cave of the Winds,” NASA SP-2014-614, 2014.

${ }^{4}$ Polhamus, E. C., "Vortex Lift Research: Early Contributions and Some Current Challenges," NASA CP-2416, Vol. 1, pp 130, Jul 1986.

${ }^{5}$ Jones, R. T., "Properties of Low-Aspect-Ratio Pointed Wings at Speeds Below and Above the Speed of Sound," NACA R835, 1946.

${ }^{6}$ Legender, R., "Ecoulement au voisinage de la pointe avant d'une aile a forte fleche aux incidences moyennes," Rech. Aero., Vol. 30, Nov-Dec 1952.

${ }^{7}$ Brown, C. E., and Michael, W. H. Jr., "Effect of Leading-Edge Separation on the Lift of a Delta Wing," Journal of the Aeronautical Sciences, Oct. 1954.

${ }^{8}$ Mangler, K. W., and Smith, J. H. B., "A Theory of the Flow Past a Slender Delta Wing With Leading Edge Separation," Proc. Roy. Soc. (London), series A., Vol. 251, No. 1265, May 1959, pp. 200-217. 1966

${ }^{9}$ Smith, J. H. B., "Improved Calculations of Leading-Edge Separation From Slender Delta Wings,” R.A.E. TR 66070, Mar.

${ }^{10}$ Polhamus, E. C., "A Concept of the Vortex Lift of Sharp-Edged Delta Wings Based on a Leading-Edge Suction Analogy”, NASA TN D-3767, Dec 1966.

${ }^{11}$ Gersten, K.: Calculation of Non-Linear Aerodynamic Stability Derivatives of Aeroplanes. AGARD Rept. 342, Apr. 1961

${ }^{12}$ Polhamus, E. C., "Application of the Leading-Edge Suction Analogy of Vortex Lift to the Drag Due to Lift of Sharp-Edge Delta Wings", NASA TN D-4739, Aug 1968.

${ }^{13}$ Lamar, J. E., “A Modified Multhopp Approach for Predicting Lifting Pressures and Camber Shape for Composite Planforms in Subsonic Flow,” NASATN D-4427, 1968.

${ }^{14}$ Wagner, S., “On the Singularity Method of Subsonic Lifting-Surface Theory,” AIAA Paper No. 69-37, Jan. 1969.

${ }^{15}$ Polhamus, E. C., "Charts for Predicting the Subsonic Vortex Lift Characteristics of Arrow, Delta, and Diamond Wings", NASA TN D-6243, Apr 1971.

${ }^{16}$ Margason, R. J., and Lamar, J. E., "Vortex-Lattice FORTRAN Program for Estimating Subsonic Aerodynamic Characteristics of Complex Planforms," NASA TND-6142, 1971.

${ }^{17}$ Lamar, J. E., and Gloss, B. B., "Subsonic Aerodynamic Characteristics on Interacting Lifting Surfaces With Separated Flow Around Sharp Edges Predicted by a Vortex-Lattice Method," NASA TN D-7921, 1975.

${ }^{18}$ Lamar, J. E., "Extension of Leading-Edge Suction Analogy to Wings with Separated Flow Around the Side Edges at Subsonic Speeds," NASA TR R-428, 1974.

${ }^{19}$ Fox, C. H. Jr., and Huffman, J. K., "Calibration and Test Capabilities of the Langley 7- by 10-Foot High-Speed Tunnel," NASA TM X-74027, 1977.

${ }^{20}$ Gloss, B. B., and Johnson, F. T., "Development of an Aerodynamic Theory Capable of Predicting Surface Loads en Slender Wings with Vortex Flow," Proceedings of the SCAR Conference, NASA CP-001, 1976, pp. 55-67

${ }^{21}$ Smith, C. W., Ralston, J. N., and Mann, H. W., “Aerodynamic Characteristics of Forebody and Nose Strakes Based on F-16 Wind Tunnel Test Experience,” NASA CR-3053, 1979.

${ }^{22}$ Luckring, J. M., “Aerodynamics of Strake-Wing Interactions,” AIAA Journal of Aircraft, Vol. 16 No. 11, 1979

${ }^{23}$ Busemann, A., “Aerodynamicher Auftrieb bei Uberschallgeschwindigkeit,” Luftfahrtforschung, Bd 12, Nr 6, Oct 1935, pp. 210-220.

${ }^{24}$ Jones, Robert T., “Wing Planforms for High Speed Flight,” NACA Rep. 863, Jun 1945. 

1981.

${ }^{25}$ Polhamus, E. C., and Toll, T. A., "Research Related to Variable Sweep Aircraft Development," NASA TM-83121, May

${ }^{26}$ Alford, W. J., and Polhamus, E. C., "Variable Sweep Wing Configuration,” United States Patent 3053484, United States Patent Office, 1962

${ }^{27}$ Polhamus, E. C., “Variable Sweep Aircraft Wing,” United States Patent 3104082, United States Patent Office, 1963.

${ }^{28}$ Polhamus, E. C., and Hammond, A. D., "Variable Sweep Wing Aircraft," United States Patent 3270989, United States Patent Office, 1966

${ }^{29}$ Gephart, V., “Airplane,” United States Patent 1524352, United States Patent Office, 1925.

${ }^{30}$ Margoulis, W., “A New Method of Testing Models in Wind Tunnels," Comps. Rendus Acad. Sci., Vol 171, Nov. 1920.

${ }^{31}$ Smelt, R., "Power Economy in High-Speed Wind Tunnels by Choice of Working Fluid and Temperature," RAE Report Aero 2081, Aug 1945.

${ }^{32}$ Polhamus, E. C., Kilgore, R. A., Adcock, J. B., and Ray, E. J., "The Langley High Reynolds Number Wind-Tunnel Program," AIAA Aeronautics and Astronautics, Oct. 1974.

${ }^{33}$ Kilgore, R. A., "The Cryogenic Wind Tunnel for High Reynolds Number Testing," Southampton University, England, Ph. D. thesis, Feb. 1974. See also NASA TM-X-70207.

${ }^{34}$ Goodyer, M. J., and Kilgore, R. A., "High-Reynolds-Number Cryogenic Tunnel," AIAA Journal, Vol. 11, No. 5, May 1973. See also AIAA 72-995, Sept. 1972.

${ }^{35}$ Adcock, J. B., "Real Gas Effects Associated with One-Dimensional Flow of Cryogenic Nitrogen," NASA TN D-8274, Dec 1976.

${ }^{36}$ Hall, R. M., "Onset of Condensation Effects with a NACA 0012-64 Airfoil Tested in the Langley 0.3-Meter Transonic Cryogenic Tunnel," NASA TP-1385, Apr 1979.

${ }^{37}$ Polhamus, E. C., “The Second Generation of Cryogenic Tunnels,” AIAA Aeronautics and Astronautics, Oct. 1981.

${ }^{38}$ Polhamus, E. C., “Applying Slender Wing Benefits to Military Aircraft,” AIAA Journal of Aircraft, Vol. 21, No. 8, Aug. 1984. See also AIAA Paper 83-2556, Oct 1983. 